\title{
Cosmic rays from microquasars: A narrow component to the CR spectrum?
}

\author{
S. Heinz ${ }^{1}$ and R. Sunyaev ${ }^{1,2}$ \\ 1 Max-Planck-Institut für Astrophysik, Karl-Schwarzschild-Str. 1, 85741 Garching, Germany \\ 2 Space Research Institute (IKI), Profsouznaya 84/32, 117810 Moscow, Russia
}

Received 18 September 2001 / Accepted 16 April 2002

\begin{abstract}
We propose that relativistic Galactic jets like those observed in GRS 1915+105 and GRO J1655-40 may produce a small but measurable contribution to the cosmic ray (CR) spectrum. If these jets contain cold protons and heavy ions (as in the case of SS433), it is plausible that this component will consist of a narrow spectral feature, with a mean particle energy corresponding roughly to the bulk kinetic particle energy in the beam, $\Gamma_{\mathrm{jet}} m_{\mathrm{p}} c^{2}$. Based on the current estimates of $\Gamma_{\mathrm{jet}}$, this feature will fall into the range of 3-10 GeV. The presence of several sources with different $\Gamma_{\text {jet }}$ will lead to the superposition of several such peaks. In addition to the narrow peaks, diffusive particle acceleration should also produce a powerlaw, whose low energy cutoff at or above $\Gamma_{\text {jet }}^{2} m_{\mathrm{p}} c^{2}$ would be visible as an additional spectral feature. The large metallicities measured in several binary companions of jet sources suggest that this CR component could have an anomalous composition compared to the bulk Galactic CR spectrum. We provide estimates of the effects of adiabatic losses which are the greatest challenge to models of narrow band CR production in microquasar jets. While the total energy contained in the microquasar CR component is highly uncertain, the local CR spectrum in the vicinity of any microquasar should be severely affected. The upcoming AMS 02 experiment will be able probe the low energy CR spectrum for such components and for composition anomalies. The spectrally peculiar gamma-ray emission produced by interaction of the ISM with CRs surrounding microquasars might be detectable by GLAST. If the presence of a microquasar CR proton component can be ruled out observationally, this argument could be turned around in favor of electron-positron jets. We show that existing OSSE/GRO and future INTEGRAL data on the Galactic $511 \mathrm{keV}$ line flux put interesting constraints on the particle content of microquasar jets. The process of CR production in relativistic flows inside the Galaxy is fundamentally different from the standard picture of CR production in nonrelativistic shocks in supernova remnants, because the particles injected by a relativistic flow are already relativistic, without any need for diffusive acceleration.
\end{abstract}

Key words. acceleration of particles - ISM: cosmic rays - ISM: jets and outflows - shock waves - black hole physics gamma rays: theory

\section{Introduction}

During the past decade, a new class of relativistic jet sources has been discovered in the Galaxy: Galactic superluminal radio sources (or microquasars), exemplified by the prototypical sources GRS 1915+105 and GRO J1655-40. Radio outbursts from these transient $\mathrm{X}$-ray binaries, containing accreting black holes or neutron stars, are associated with ejections of plasma at relativistic bulk velocities. The bulk Lorentz factors of these flows inferred from observations are of the order of $\Gamma_{\text {jet }} \sim 3-10$, though exact observational determination of $\Gamma_{\text {jet }}$ is difficult and in any case it is unlikely that a universal value of $\Gamma_{\text {jet }}$ holds for all jets. For reasons of simplicity and for lack of better knowledge we will adopt a fiducial value of $\Gamma_{\text {jet }} \sim 5$ for numerical examples, with the explicit understanding that Galactic jets most likely operate at a variety of Lorentz factors, which are currently not well determined. These ejections can

Send offprint requests to: $\mathrm{S}$. Heinz,

e-mail: heinzs@mpa-garching.mpg.de contain energies in excess of $10^{44}$ ergs (Mirabel \& Rodríguez 1994; Hjellming \& Rupen 1995; Fender et al. 1999a), and they occur at a Galactic rate of $\gtrsim f e w \mathrm{yr}^{-1}$.

A large part of the kinetic energy transported by these jets is transferred into random, isotropic particle energy at the interface between the jet and the ambient medium, the working surface. Because the jets are relativistic, the particles leaving the working surface must a priori be relativistic without any need for diffusice acceleration. This mechanism of accelerating relativistic cosmic ray $(C R)$ particles is fundamentally different from CR production in the non-relativistic shocks of supernova remnants (SNRs), which provides the bulk of the Galactic CRs.

While the momentum gain for particles crossing a nonrelativistic shock is small (of order $\delta p / p \sim v / c$ ), the large momentum gains encountered in relativistic shocks (of order $\delta p / p \sim \Gamma$, where $\Gamma$ is the shock Lorentz factor) should lead to the formation of distinct spectral features in the spectrum (see Sect. 3.3.1). Thus, unlike the CRs produced in SNRs, which follow a smooth powerlaw spectrum, the CRs produced 


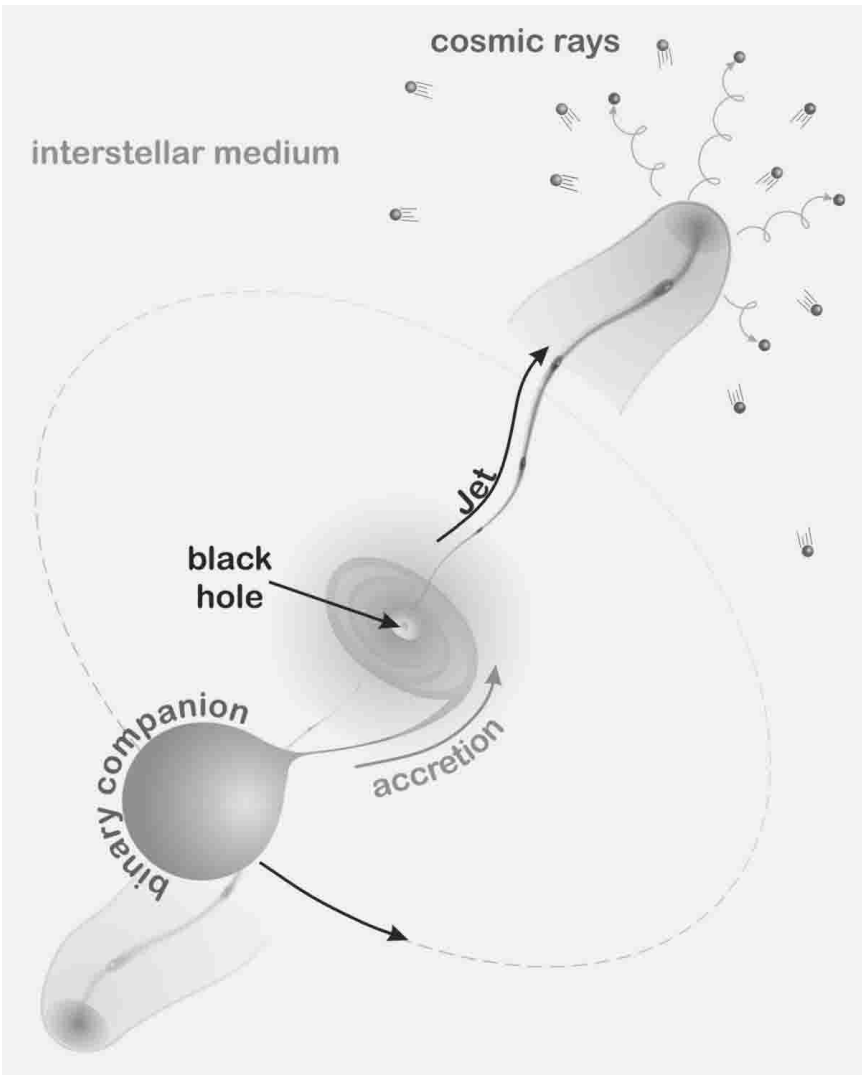

Fig. 1. Cartoon of the proposed model of CR production in microquasars: The interface between the relativistic jet and the ISM is a natural site for the production and release of relativistic particles.

in relativistic flows, like those encountered in microquasars, should show clearly distinguishable, and possibly narrow, spectral features.

If these particles can escape the working surface without suffering significant adiabatic energy losses, they will diffuse through interstellar space, and will thus contribute to the Galactic cosmic ray (CR) spectrum ${ }^{1}$.

Based on the arguments presented in this paper, we conclude that an additional component of CRs generated by relativistic jets in microquasars should exist in the Galaxy. Initially, this component should consist of narrow peaks, with peak energies corresponding to $\Gamma_{\text {jet }} m_{\mathrm{p}} c^{2}$ from different jet sources.

There are many mechanisms which might broaden these features. However, any observational limits on their existence would give us additional information about the physics of microquasar jets and the physical conditions in relativistic shocks. Below, we will discuss the main mechanisms which could smooth out the component under discussion. In this paper, we

\footnotetext{
1 The production of ultra-high energy CRs in Galactic X-ray binaries has been discussed in the past in the context of Cyg X-3 (Lloyd-Evans et al. 1983; Manchanda et al. 1993; Mitra 1994), though recent studies have not confirmed the presence of ultra-high energy gamma rays (Borione et al. 1997). This scenario is shown as a cartoon in Fig. 1. The production of CRs in microquasars has so far only received minor attention in the literature (for example, it was mentioned as an alternative source to produce all CRs by Dar \& De Rújula 2001), and requires further study.
}

content ourselves with presenting order of magnitude estimates only, since the goal of the paper is to point out to the CR community that, in addition to $\mathrm{CR}$ acceleration in supernova remnants (SNRs), there is another very effective mechanism to release relativistic particles in the Galaxy. Traces of these particles might be hidden in the observed CR spectra, in $\gamma$-rays with energies of a few $100 \mathrm{MeV}$, and possibly in electron-positron annihilation line emission from regions close to the location of microquasars in the Galactic plane.

\section{Outline of the model}

In this section we will present a general description of the model proposed for $\mathrm{CR}$ production in microquasars.

As we will argue below, it is likely that the plasma traveling far downstream in the jet towards the working surface is cold (the mean particle velocity is $\left\langle v^{2}\right\rangle \ll c^{2}$ in the rest frame of the jet plasma), especially if microquasar jets are composed of electron-ion plasma. The same is, of course, also true for the undisturbed interstellar medium (ISM). Thus, the bulk of the plasma transported to the interface between the jet and the ISM (for simplicity we will call this interface the working surface of the jet, regardless of its detailed physical structure) is initially cold.

This conjecture is inspired by observations of the mildly relativistic jets in SS433 (the best studied relativistic Galactic jet to date, albeit mildly relativistic and not considered a microquasar). In this source, red- and blue-shifted Balmer $\mathrm{H} \beta$ and other optical recombination lines, usually radiated by plasmas with temperatures of order $10^{4} \mathrm{~K} \sim 1 \mathrm{eV}$, allow the determination of the bulk velocity of the flow: $0.26 c$. This velocity is remarkably constant over the 20 years the source has been observed (Margon 1984; Milgrom et al. 1982). ASCA (Kotani et al. 1998) and recent Chandra (Marshall et al. 2002) observations of X-ray lines of hydrogen- and helium-like ions of iron, Argon, Sulfur, and Oxygen show that these ions are moving in the flow with the same velocity, $0.26 c$. This X-ray emitting plasma at temperatures of $T \sim 10^{7}-10^{8} \mathrm{~K} \sim 10^{3}-10^{4} \mathrm{eV}$ is observed at much smaller distances $\left(\sim 10^{11} \mathrm{~cm}\right)$ from the central compact object than the optical line emission region $\left(\sim 10^{14} \mathrm{~cm}\right)$. A striking feature of the SS433 jet is that the plasma is observed to be moving with relativistic velocities. Yet, at the same time the jet plasma itself shows very little line broadening (i.e., it is cold).

If microquasar jets are similar to the SS433 jets in composition and properties (i.e., cold electron-ion plasma at relativistic bulk speeds) the consequences for the interpretation of these jets will be far reaching, as we will argue below. Independent from this argument, the radio synchrotron emission detected from microquasar jets and several radio nebulae surrounding microquasar sources (see Sect. 3.1) is clear evidence for the presence of relativistic electrons, which, when released into the ISM, will act as cosmic ray electrons.

\subsection{The standard picture for jet working surfaces}

The standard picture for the interface between powerful radio galaxies and their environment is a strong double shock 


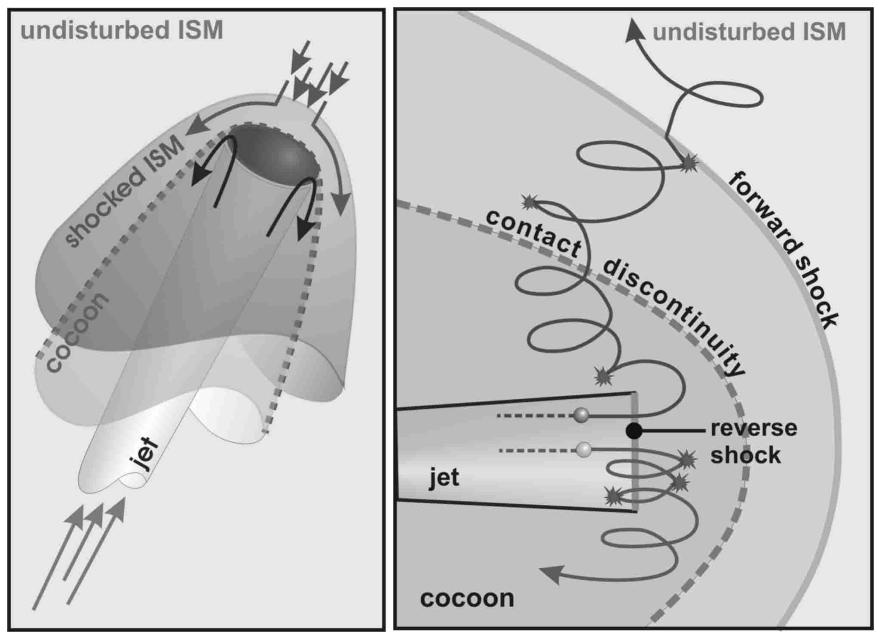

Fig. 2. Cartoon of the standard picture of the interface between jet and ISM (left panel), as envisaged to apply in FR II radio galaxies. The injection of relativistic particles can occur either in the reverse or forward shock. Right panel: cartoon of particle trajectories for particles crossing the shock only once (upper solid line) and particles participating in diffusive shock acceleration (lower solid line), particle scattering indicated as stars.
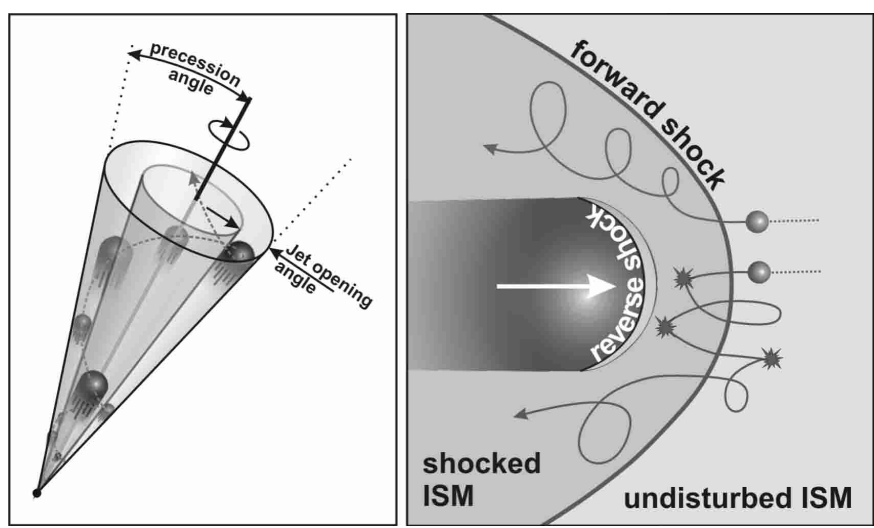

Fig. 3. Left: cartoon of a jet composed of discrete ejections with precession. In such a non-stationary picture, each ejection is slowed by its interaction with the ISM (which might be disturbed by previous ejections). This interaction will likely happen in the form of a forward shock (into the ISM). ISM particles will leave the shock with energies of order $\Gamma_{\text {jet }} m_{\mathrm{p}} c^{2}$ (here, $\Gamma_{\mathrm{jet}}$ is the Lorentz factor of the individual ejection, which decreases with time). Right: cartoon of particles crossing a relativistic forward shock, likely the appropriate scenario for a cold ejection running into the ISM. Same nomenclature as in Fig. 2.

structure (forward shock into the ISM and reverse shock into the jet), shown in Fig. 2. The shocked jet material is shed at the head of the jet and inflates an enshrouding cocoon around the jet, filled with relativistic plasma, which has gone through the terminal shock. Such a scenario might also be relevant for the terminus of Galactic relativistic jets. A similar picture arises if the jets are composed of discrete ejections, propagating into an external medium at relativistic speeds, as sketched in Fig. 3.

A cold upstream particle crossing an ultra-relativistic shock into a downstream region with relative Lorentz factor $\Gamma_{\text {rel }} \sim$ $\Gamma_{\text {jet }}$ will have an internal energy of $\gamma m c^{2} \sim \Gamma_{\text {jet }} m c^{2}$ in the downstream frame after the first shock crossing. Consequently, all initially cold particles will leave the shock with about the same specific energy $\Gamma_{\text {jet }} c^{2}$. Particles can pick up additional energy if they cross the shock multiple times, which is the basis of diffusive shock acceleration schemes like Fermi acceleration, resulting in the formation of a powerlaw distribution. However, as has recently been shown by Achterberg et al. (2001), the bulk of the particles crossing a relativistic shock escape after the very first shock passage and will therefore not participate in diffusive shock acceleration. It is these particles that carry off the bulk of the dissipated jet energy.

As a result, the bulk of the particles might leave the shock with a narrow energy distribution, peaking at an energy close to the specific kinetic energy of the jet: $\left\langle\gamma m c^{2}\right\rangle \sim \Gamma_{\text {jet }} m c^{2}$, with an energy width similar to or higher than the Lorentz transformed thermal velocity, $\Delta \gamma \sim 2 \gamma c_{\mathrm{s}}$ (i.e., very narrow, since the internal sound speed $c_{\mathrm{s}}$ is small: $\left.c_{\mathrm{s}} \ll 1\right)$.

Whether this narrow distribution will be preserved as the particles travel away from the shock, or whether it will be thermalized, depends on the efficiency of collective plasma effects and small angle scattering on magnetic field irregularities, which are also needed to isotropize the particle distribution. If collective effects are strong, the particle spectrum will be broadened into a relativistic Maxwell-Boltzmann distribution, with a temperature corresponding to the value given by the relativistic Rankine-Hugoniot jump conditions. In the case of a strong, ultra-relativistic shock, this is simply $k T \sim 1 / 3 \Gamma_{\text {jet }} m c^{2}$, i.e., the mean particle energy is just $\Gamma_{\text {jet }} m c^{2}$ (e.g. Blandford \& McKee 1976). In this case the relativistic proton plasma in the shocked ISM is equivalent to the X-ray emitting gas in SNR shocks. However, in microquasar shocks we have extremely rarefied, relativistic particles with a relatively narrow thermal (i.e., not powerlaw) energy distribution.

\subsection{Termination without strong shocks?}

However, the structure of relativistic shocks is still not well understood and it might be that this interface is not a simple double shock structure. It could be significantly different in nature. For example, the jet could be magnetically connected with the environment, i.e., if the flux tubes join smoothly with the large scale magnetic field of the ISM, as shown in the cartoon in Fig. 4 (note, however, that Lubow et al. 1994 showed that realizing such configuration is rather difficult).

In such a case the shock would be replaced by stochastic pitch angle scattering of the particle distribution (this can occur if the jet is moving sub-Alfvénically, for example). Since the jet plasma is traveling relative to the ISM, such a scenario would excite strong two-stream instabilities at the interface between ISM and jet plasma, which would isotropize and possibly thermalize the particle distribution of the jet very quickly. The deposition of jet thrust would then imply that this interface is itself moving through space. Precession, as observed in SS433 (e.g., Milgrom 1979) and suggested to be present in GRO 1655-40 (Hjellming \& Rupen 1995), will significantly alter the dynamical balance between ISM and jet plasma, as will the time dependent nature of the interface if the jets are composed of discrete ejections. 


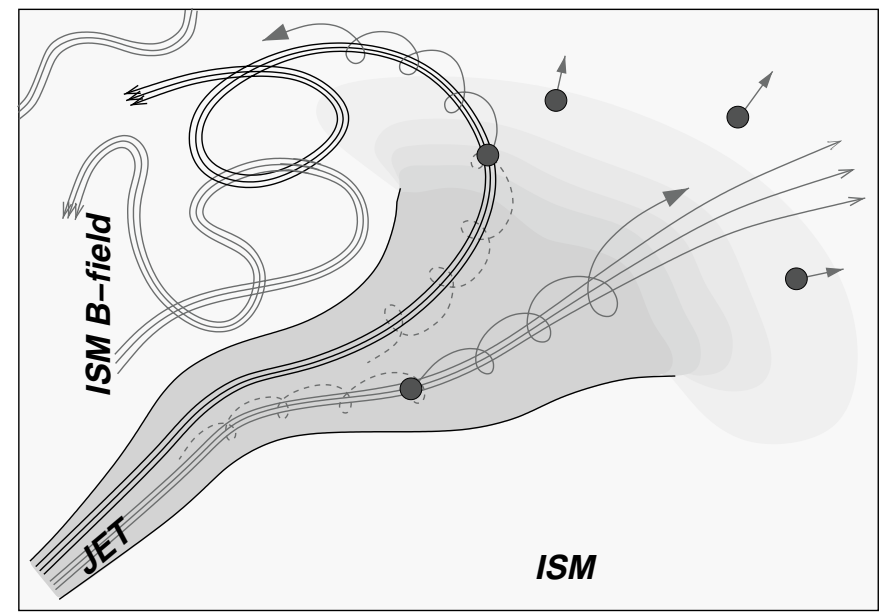

Fig. 4. Cartoon of particle injection by a jet without a strong shock at its interface with the ISM (e.g., if the magnetic structure of the jet is connected with the ISM and the jet is sub-Alfvénic). Dots represent CRs transported by the jet and released into the ISM at the bulk speed of the jet, grey arrows represent magnetic flux tubes. The right flux tube representation depicts the case where adiabatic losses are important, the left shows a case where the field flux tube is smoothly fused with the stochastic interstellar field and adiabatic losses might not be dominant in particle transport.

If furthermore the magnetic field is stochastically tangled on small scales, the detailed behavior of the plasma could be very complicated, with a gradual change from relativistic, ballistic motion to random propagation. Qualitatively, this would be comparable to extragalactic FR I sources (though the exact nature of the dynamics in FR I sources is not yet clear, either).

In such a case, the absence of a strong shock would preclude diffusive shock acceleration (though stochastic acceleration might still exist if particles scatter off of relativistic turbulence which might exist in the transition region between jet and ISM). Only the narrow or thermalized component with mean energy of $\Gamma_{\text {jet }} m_{\mathrm{p}} c^{2}$ and strong cutoff at higher energies would exist.

\subsection{Spectral characteristics}

Based on Sects. 2.1 and 2.2, we therefore predict that each Galactic microquasar produces a narrow component of CRs, which peaks at an energy $\Gamma_{\text {jet }} m c^{2}$. For protons, this energy should fall into the range of $\Gamma_{\text {jet }} m_{\mathrm{p}} c^{2} \sim 3-10 \mathrm{GeV}$. The superposition of these spectral signatures from several microquasars will appear as a broad feature in the energy range from 3-10 GeV.

Even if most of the particles are thermalized downstream, the spectrum will still show a steep turnover beyond energies of order $3 k T \sim \Gamma_{\text {jet }} m_{\mathrm{p}} c^{2}$ (see the dashed curve in Fig. 5), which will appear as an edge-like feature in the overall CR spectrum.

Similarly, a number of other processes will tend to broaden any narrow component produced in the working surface, including adiabatic losses (competing with diffusion of particles out of the loss region, see Appendix B and the right panel in Fig. 5) and solar modulation. The effect of these processes will be to spread particles to lower energies, leaving the strong turnover/cutoff above energies of $\Gamma_{\text {jet }} m c^{2}$ intact.

The only serious cooling these CR protons at energies of a few $\mathrm{GeV}$ might experience will be adiabatic losses, which will occur if the particles are confined to an expanding plasma volume (e.g., if it is overpressured with respect to the environment). However, since many processes can lead to increased diffusion of these particles, it appears plausible that a large fraction of the CRs might escape before they suffer strong adiabatic losses.

If a component of cold electrons is also present in the jets in addition to the observed powerlaw electrons, a similar, very low energy relativistic electron component (around 2-5 MeV) might appear. However, it would contain only a fraction $m_{\mathrm{e}} / m_{\mathrm{p}}$ of the energy in the proton component.

The remaining fraction of particles (both ions and electrons) which do not escape the shock after the first shock passage and thus perform multiple shock crossings will be accelerated diffusively to a powerlaw-like distribution. Only the high energy tail of this powerlaw-like electron component is directly observable via synchrotron radio emission.

Likening the acceleration of particles crossing a relativistic shock to the problem of Compton up-scattering of low energy photons on relativistic thermal electrons (see, for example, Pozdnyakov et al. 1983), we note that a particle scattered both up-stream and down-stream of the shock will experience an energy gain by a factor of order $\Gamma_{\text {rel }}^{2}$ per crossing cycle, where $\Gamma_{\text {rel }} \sim \Gamma_{\text {jet }}$ is the relative Lorentz factor between upstream and downstream plasma. This was argued by Vietri (1995), applied to the acceleration of particles in gamma-ray burst shocks. This will lead to the production of several peaks in the spectrum. The input spectrum for this up-scattering process is the narrow particle population produced in the initial shock crossing (discussed above), and thus peaks will appear at energies $\sim f e w \times \Gamma_{\text {jet }}^{2 i+1} m_{\mathrm{p}} c^{2}$, where $i$ is the number of shock crossing cycles performed by the particle. The normalization of each peak, and thus the approximate powerlaw index, is determined by the escape probability of the particles (similar to the optical depth in inverse Compton scattering). The resulting spectrum is sketched in Fig. 5.

Note, however, that Achterberg et al. (2001) argue that higher order shock crossings do not lead to energy gains of order $\Gamma_{\text {rel }}^{2}$. In their treatment, scattering is limited to very small angles and the energy gain is only of order unity, and thus the position of the peaks would be much more closely spaced, resembling a powerlaw much more than in the Compton scattering analogy discussed in the previous paragraph. The low energy turnover (or cutoff) of this powerlaw distribution would then be located roughly at $\Gamma_{\text {jet }}^{2} \sim 10-100 \mathrm{GeV}$. At higher energies, multiple scattering will form a powerlaw with index ${ }^{2}$ $s \sim 2.3$. According to this simple approach, the difference between these two pictures is therefore the energy of the second peak $\left(\sim \Gamma_{\text {jet }}^{3}\right.$ vs. $\left.\sim \Gamma_{\text {jet }}^{2}\right)$.

\footnotetext{
${ }^{2}$ Note that powerlaw indices from cosmic ray modified shocks tend to be steeper at least near the low energy cutoff (e.g., Berezhko \& Ellison 1999; Ellison \& Double 2002).
} 

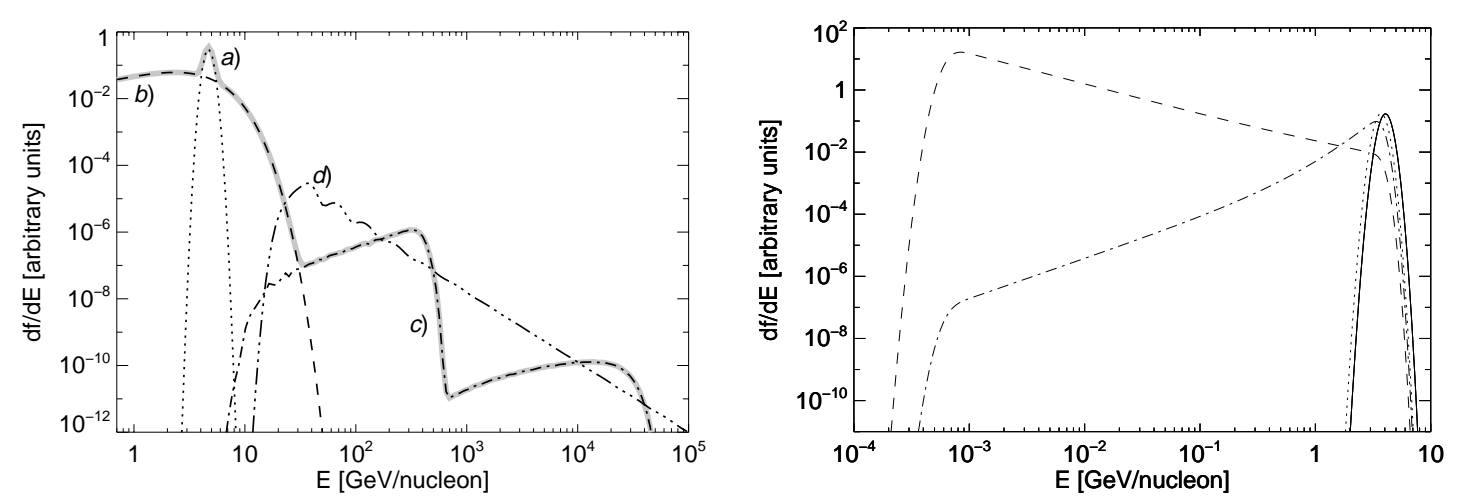

Fig. 5. Left panel: sketch of the predicted contribution from a microquasar to the Galactic CR spectrum for $\Gamma_{\text {jet }}=5$. a) Dotted line: narrow component, corresponding to cold $\left(T \sim 7 \times 10^{10} \mathrm{~K}\right)$ upstream particles simply isotropized upon crossing the working surface (Lorentz transformed energy distribution), arbitrary normalization; $b$ ) dashed line: Maxwell-Boltzmann component from particles thermalized in the shock (normalized to contain the same power as component $a$ ); $c$ ) dash-dotted line: powerlaw-like component (escape probability $99 \%$, normalized to contain $10 \%$ of component $a$ ) for the case of strong scattering. Note the similarity to Comptonization of low energy seed photons by a relativistic medium with low optical depth (i.e., high escape probability); d) dash-triple-dotted line: powerlaw spectrum for weak scattering case (Achterberg et al. 2001) for the same normalization as component $c$ ). Note the difference in the position of the first peak between $c$ ) and d), $\sim \Gamma_{\text {jet }}^{2}$ vs. $\sim \Gamma_{\text {jet }}^{3}$. Right panel: estimate of the effects of adiabatic losses on the particle distribution for different ratios of the adiabatic losses time $\tau_{\text {ad }}$ of the particles to the escape time $\tau_{\text {esc }}$, compared to the injected particle distribution (solid) line for a pressure differential between shock and ISM of $p_{\text {shock }} / p_{\text {ISM }}=3 \times 10^{8}$ (see Appendix B): strong adiabatic losses $\left(\tau_{\text {ad }} / \tau_{\text {esc }}=10^{-3}\right.$, dashed line), intermediated losses $\left(\tau_{\text {ad }}=\tau_{\text {esc }}\right.$, dash-dotted line), and weak adiabatic losses $\left(\tau_{\mathrm{ad}} / \tau_{\mathrm{esc}}=10^{3}\right.$, dotted line $)$.

Since the structure of relativistic shocks, and their presence in the working surfaces of microquasar jets are subject to considerable uncertainty, the observational discovery of any of the features discussed in this paper (and in particular the second peak, which would help to distinguish between the two scenarios of diffusive acceleration mentioned in the previous paragraphs, see Fig. 5) or evidence of their absence would be important input into theories of relativistic shocks.

\subsection{Comparison with the canonical $C R$ powerlaw component}

The CR components described above and shown in Fig. 5 should be compared to the well known powerlaw CR component observed near earth: CR protons and nuclei have a powerlaw spectrum with a uniform slope around $s \sim 2.5-2.7$ over an extremely broad energy range from $1 \mathrm{GeV}$ up to $10^{15} \mathrm{eV}$. Locally, the CR energy density is of order $10^{-12} \mathrm{ergs} \mathrm{cm}^{-3}$. Observations of the light elements produced by spallation reactions show that the lifetime $\tau_{\mathrm{CR} \text {, disk }}$ of relativistic protons in the Galactic disk is of order $\tau_{\mathrm{CR} \text {, disk }} \sim 1.5 \times 10^{7} \mathrm{yrs}$ (Yanasak et al. 2001), while the lifetime in the Galactic halo $\tau_{\mathrm{CR} \text {, halo }}$ is close to $10^{8}$ years (e.g., Ginzburg 1996). This enables us to estimate the CR luminosity of the Galaxy as $L_{\mathrm{CR}} \sim 4 \times 10^{40} \mathrm{ergs} \mathrm{s}^{-1}$, with a diffusion coefficient $\kappa$ inside the disk of

$\kappa \sim \frac{H_{\text {disk }^{2}}}{\tau_{\mathrm{CR}, \text { disk }}}=2 \times 10^{28} \frac{\mathrm{cm}^{2}}{s} \frac{H_{\mathrm{kpc}}^{2}}{\tau_{15}} \sim 10^{28.3} \frac{\mathrm{cm}^{2}}{\mathrm{~s}} \kappa_{28.3}$

where $H_{\mathrm{kpc}}$ is the disk height in kpc, $\tau_{15}$ is the CR lifetime in the disk in units of 15 Myrs, and $\kappa_{28.3}$ is the ISM diffusion coefficient, normalized to a value of $2 \times 10^{28} \mathrm{~cm}^{2} \mathrm{~s}^{-1}$. The current paradigm for the bulk of the CRs observed in the vicinity of the earth is that they are produced by shock acceleration in the decelerating blast waves of SNRs (e.g. Blandford \& Ostriker 1978). The usual assumption is that about $5 \%$ of the mechanical energy of the SNR are converted into CR energy.

There is no doubt that in the vicinity of an active microquasar the low energy part of the Galactic CR spectrum must be strongly distorted. As a result, smooth maxima or edge-like features should exist in the few $\mathrm{GeV}$ range of the CR spectrum. For a distant observer, the signals from several sources will be superimposed due to the long diffusion time through the galaxy. Integrally, though, deviations from the powerlaw spectrum expected in diffusive shock acceleration models should be observable.

Energy estimates which we present below show that this $\mathrm{CR}$ component produced in microquasars might contribute measurably to the spectrum of the CR protons in the energy band mentioned above. We will argue that, globally, microquasars should contribute upward of $0.1 \%$ of the total Galactic CR power. However, the locally measured (i.e., near earth) relative strength of the proposed $\mathrm{CR}$ components produced in microquasars compared to the canonical CR powerlaw distribution is highly uncertain, as it depends on the history of microquasar activity in our Galactic neighborhood.

Given these uncertainties, it might be rather difficult to detect the tiny deviations in the CR spectrum caused by distant microquasars (further complicated by the strong effects of solar modulation at and below the predicted energy range). However, they might be measurable by the upcoming $A M S 02$ experiment (e.g. Barrau 2001), which will offer unprecedented sensitivity and will be launched during the upcoming solar minimum (reducing the effects of solar modulation significantly). Traces of such a component might also be present in already existing high quality data sets from past or ongoing experiments, such as IMAX (Menn et al. 2000) or CAPRICE (Boezio et al. 1999). 
Absence of any traces of spectral deviations in the upcoming AMS 02 experiment might become a strong argument in favor of electron-positron jets in Galactic superluminal radio sources or, alternatively, it would demonstrate that there is an unknown acceleration mechanism with $100 \%$ efficiency of transforming of the mechanical beam energy into a relativistic powerlaw distribution. Given these premises, we can state that one of the following two statements must hold: 1) either an additional hadronic CR component exists (though it may be so weak that detection inside the solar system is impossible) or 2) all jets are electron-positron dominated (in which case an additional CR electron-positron component should exist).

\subsection{Peculiar abundances}

Recent studies of the chemical composition of Galactic transients indicate that the binary companions of several microquasar sources are metal enriched: the heavy element abundances (e.g., $\mathrm{N}, \mathrm{O}, \mathrm{Ca}, \mathrm{Mg}$ ) in the optical counterparts of the X-ray sources GRO J1655-40 (Israelian et al. 1999) and V4641 Sgr (Orosz et al. 2001) exceed solar abundances by about an order of magnitude, much more so than the observed overabundance of the same elements in CRs (Zombeck 1990). Furthermore, the relative abundances between these elements are unusual compared to either solar abundances or the abundance pattern observed in the bulk of the CR spectrum.

These abundance anomalies in GRO J1655-40 and V4641 Sgr could be the result of mass exchange between two rapidly evolving massive stars or enrichment of the normal stellar atmosphere during the supernova explosion of the primary predecessor. Accretion brings these abundance anomalies into the jet creation region in the inner disk, from where they could be transported out by the jet, eventually producing CRs by the mechanism outlined above. Similarly, Cyg X-3 is known to have an extremely hydrogen deficient Wolf-Rayet companion (van Kerkwijk et al. 1992; van Kerkwijk et al. 1996; Fender et al. 1999b), which could also lead to a large overabundance in helium and heavier elements relative to hydrogen in the produced CR spectrum.

Therefore, Galactic jets might be responsible for part of the observed CR abundance anomalies. Moreover, the CR component produced in relativistic jet sources inside the Galaxy might show rather unusual chemical abundances, in comparison with the bulk of the CRs in the powerlaw population. This would immediately distinguish Galactic jets from other CR creation mechanisms. The comparison between the measured abundances in the energy range where we expect Galactic jet sources to contribute (of order a few $\mathrm{GeV}$ ) with those measured in the pure powerlaw regime will thus be an important probe to search for the proposed CR component. Note that the CRs produced in SNRs originate in the external shock of the swept up ISM, thus the abundances of the produced CR spectrum reflect the ISM, which might have been enriched by a pre-collapse wind, but will not show the peculiar abundance of the SN ejecta. Because all the spectral components accelerated in microquasars originate from the same plasma, they should all show the same abundance pattern. This could be a way to associate spectral features at different $\mathrm{CR}$ energies with a microquasar origin.

A second way to distinguish particles accelerated in the relativistic shocks of Galactic microquasars from those accelerated in non-relativistic SNR shocks is the different energyparticle mass relation: All particles in relativistic cold jets have the same Lorentz factors. Since single-pass shock acceleration will accelerate all particles to roughly the same random Lorentz factor, the peak energy for different species will be proportional to their rest mass (i.e., a fixed energy per nucleon). Electromagnetic acceleration processes would instead produce particle energies proportional to $Z / A$. This difference might again be measurable by $A M S 02$, and might already be present in CR data on heavy nuclei from experiments like $H E A O-3$ (Engelmann et al. 1990) or ACE (Binns et al. 2001).

\section{Energetics and spectra of microquasar CRs}

\subsection{Microquasar energetics}

The best studied cases of microquasar activity show that large amounts of kinetic energy can be liberated: conservative equipartition estimates of the energy released in the major outbursts of GRS $1915+105$ give $E_{\text {kin }}>2 \times 10^{44}$ ergs (Mirabel \& Rodríguez 1999; Fender et al. 1999a), released over a period of a few days at most.

Existing radio monitoring data (Pooley \& Fender 1997; Foster et al. 1996) show that GRS $1915+105$ exhibits several giant flares per year, not all of which were observed with detailed campaigns (e.g., Fender et al. 1999a). This yields an estimated average kinetic power, and, since almost all of the energy will initially be deposited in the form of CRs, an estimated cosmic ray power of $L_{\mathrm{kin}}>10^{37} \mathrm{ergs} \mathrm{s}^{-1}$ for GRS $1915+105$ alone. In fact, GRS $1915+105$ seems to release an even higher power in the form of microflares between major outbursts, estimated to exceed few $\times 10^{37} \mathrm{ergs} \mathrm{s}^{-1}$ (Mirabel \& Rodríguez 1999) and even $3 \times 10^{38} \mathrm{ergs} \mathrm{s}^{-1}$ (Fender \& Pooley 2000).

Using the publicly available GBI monitoring data (http://www.gb.nrao.edu/fgdocs/gbi/gbint.html), we estimate that GRS $1915+105$ spends in excess of $60 \%$ of its time at flux levels significantly enhanced over the baseline flux (GBI monitoring data of Cyg X-3 show a similar rate), with about 2 major outbursts per year (see Fig. 6 and also Fender et al. 1999a; Foster et al. 1996). Assuming that the observed radio flux in flares is proportional to the amount of kinetic energy released in the flare, and using the observed 1997 flare (Fender et al. 1999a) with a minimum kinetic energy estimate of $2 \times 10^{44} \mathrm{ergs}$, we estimate that over the period covered by GBI monitoring, the mean kinetic luminosity of GRS $1915+105$ in flares is of order $L_{\mathrm{kin}} \sim 10^{38} \mathrm{ergs} \mathrm{s}^{-1}$. If the baseline radio emission from GRS $1915+105$ is also due to low level jet emission, this estimate increases by a factor $\sim 1.4$.

The jets in SS433 are even more impressive: Reasonable estimates put the total, continuous kinetic power in excess of $L_{\text {kin }} \sim$ few $\times 10^{38}-$ few $\times 10^{39} \mathrm{ergs} \mathrm{s}^{-1}$ (Marshall et al. 2002; Margon 1984; Spencer 1984), which is already of order 1-10\% of the total Galactic CR luminosity. While the jets in SS433 are only mildly relativistic, and the production of observable, 


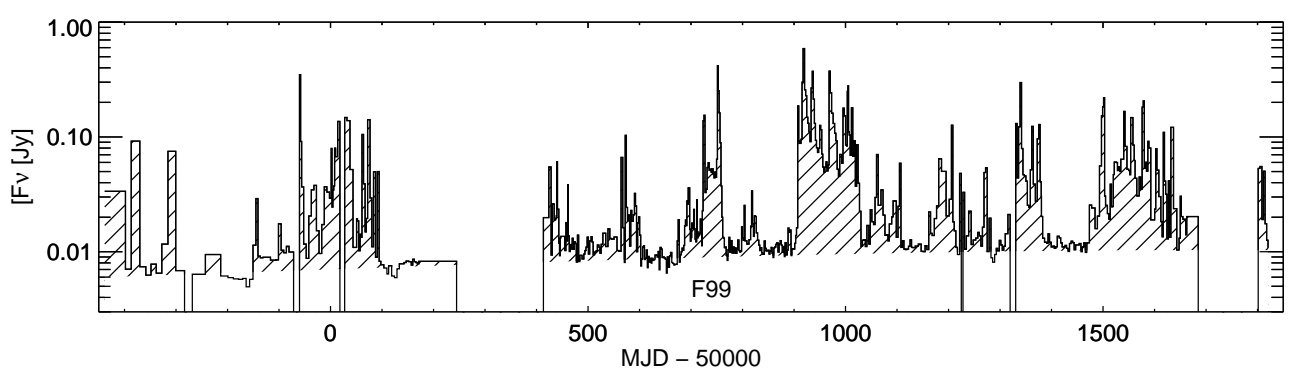

Fig. 6. Plot of the $2.25 \mathrm{GHz}$ GBI monitoring data (http://www.gb.nrao.edu/fgdocs/gbi/gbint.html) for GRS $1915+105$ over the time span from June 1994 to August 2000. Shown as a hatched area is the flux considered above the baseline flux, i.e., the flux considered to originate from flares, which we integrated to arrive at the estimate for the average kinetic power carried by the jet. The flare analyzed by Fender et al. (1999a) is indicated by the mark "F99". The large gaps are due to gaps in the monitoring campaign and were not included in the procedure.

relativistic CRs thus falls under similar restrictions with regard to particle acceleration efficiency as supernovae, the example of SS433 does show that Galactic jets are capable of releasing impressive amounts of kinetic energy. Thus, jets from objects like SS433 (with mildly relativistic bulk speeds) might be an important source of sub-CRs in the Galaxy, influencing heating and ionization of the ISM.

Since the subject of Galactic microquasars is still relatively young, and many of the known sources have only been discovered in recent years, estimating the true Galactic rate of radio outburst events and thus the total Galactic power in relativistic jets is difficult. Taking the interval from 1994 through 2000 , there were at least 7 well observed giant radio outbursts comparable in strength with GRS 1915+105 (corrected for Galactic distance) in the sources Cyg X-3 (Mioduszewski et al. 2001), GRO J1655-40 (Hjellming \& Rupen 1995), GRS 1915+105 (Mirabel \& Rodríguez 1994; Fender et al. 1999a), V4641 Sgr (Orosz et al. 2001), and XTE J1748-288 (Fender \& Kuulkers 2001), giving a very conservative lower limit on the Galactic rate of $1 \mathrm{yr}^{-1}$. As with GRS 1915+105, we expect many giant flares to have gone unnoticed, and a more reasonable estimate of the event rate would be of the order of 10-100 Galactic events per year.

Cyg X-3, which is believed to be relativistically beamed, shows radio peak luminosities up to 200 times stronger than GRS 1915+105 (Fender \& Kuulkers 2001), and often exhibits flaring activity (Ogley et al. 2001) at or above the peak level of GRS $1915+105$ on timescales of $\sim 10$ days. The other sources mentioned above are very similar to each other in peak radio luminosity (Fender \& Kuulkers 2001), which we take as an indicator of kinetic power (most of these sources are not resolved and an estimate of the equipartition energy of the jet is thus not possible).

If indeed these sources operate on the same level as GRS 1915+105, the minimum kinetic luminosity of these seven sources together would be $L_{\text {kin }} \gtrsim 10^{39} \mathrm{ergs} \mathrm{s}^{-1}$. Since this estimate is based on the minimum energy estimates of $L_{\mathrm{kin}}$ in GRS $1915+105$, the true kinetic luminosity of these sources might well be much larger.

Furthermore, there are many sources that are known to have been active at earlier epochs [e.g., V404 Cyg, Han \& Hjellming 1992 and Cir X-1, Haynes et al. 1978], which exhibited flux levels comparable to the above mentioned sources.
Many sources currently active might simply not have been detected yet. Similarly, many more X-ray sources are observed to be consistently active at lower radio fluxes (e.g., Cyg X-1, or GX 339-4, Fender 2001) than the brightest sources mentioned above.

During the past few years, it has become clear that radio emission from Galactic X-ray sources is a very common phenomenon. Radio emission is usually detected during state changes of the X-ray source (into or out of the low/hard state), including soft X-ray transients. While the powerful radio flares discussed above are associated with such transients, there are many more X-ray sources which are active at lower radio flux levels (e.g., Cyg X-1, or GX 339-4, Fender 2001).

These sources are observed to produce stationary, optically thick jet emission (as opposed to the already optically thin emission detected in typical radio flares of transient jets). It is not clear whether these jets are in fact relativistic and how much energy they carry. One might hope to estimate the kinetic power from the observed flux, scaling it to the peak flux observed in GRS 1915 as was done above for transient sources, but detailed kinematic modeling of the jet would be required to justify such a simple argument. In any event, because no complete sample of such sources exists, it is impossible to estimate the total fraction of mechanical jet power contained in low power sources. Furthermore, these sources might have shown transient activity in the past as well, given that GRS $1915+105$ also shows steady, quiescent radio emission at comparable flux levels.

Other sources like 1E 1740-294 (Mirabel et al. 1992), GRS 1758-258 (Martí et al. 1998), and Cir-X1 (Stewart et al. 1993) show permanent extended structure resembling radio lobes in extragalactic radio sources, which are witness to past radio activity and must harbor a significant amount of CRs as well. We note here that estimating the total kinetic power from the presence or absence of radio lobes in microquasar sources (indicating past activity) is severely hampered by the fact that Galactic radio lobes are expected to have very low surface brightness (Heinz 2002).

The estimate for the kinetic energy output from GRS 1915+105 (Fender et al. 1999a), which we used as a template case to estimate the total Galactic energy in jets, is based on the assumption that the jet plasma is composed of a powerlaw of relativistic electrons and cold protons (for charge conservation). The electron spectrum was assumed to extend 
only over the range in frequency observed in the radio. While an IR detection of the jet indicates a high energy tail of the spectrum (Sams et al. 1996; Mirabel et al. 1996; Eikenberry et al. 1998), a low energy component (down to $\gamma \sim 1-10$ ) has never been observed in any jet due to lack of viable emission mechanisms to reveal such a component. The possibility of detecting this component via inverse Compton scattering has been discussed, for example, by Ensslin \& Sunyaev (2001).

Finally, the physical structure of microquasar jets is still not known-they could be made of either discrete ejections or a continuous stream of matter. If the jet is not composed of discrete ejections, but instead is a continuous outflow with knots corresponding to internal shocks, Kaiser et al. (2000) have shown that, in the case of GRS $1915+105$, the estimate of the total kinetic energy carried in the jet (and thus the total CR energy released in the working surface) is a factor of 10 higher than the above estimate (though the instantaneous kinetic power is reduced), corrections for the low energy end of the particle distribution notwithstanding.

All of this indicates that the lower limit of $L_{\mathrm{kin}} \sim$ few $\times$ $10^{38} \mathrm{ergs} \mathrm{s}^{-1}$ is conservative, and it might be that the kinetic luminosity from microquasars is of the order of $10 \%$ of the total Galactic cosmic ray power $L_{\mathrm{CR}}$. Clearly, the uncertainty in frequency and power of radio flares in microquasars warrants continued monitoring of these sources to answer the question of how important energy input by these sources really is.

\subsection{The termination shock}

In the following, we will assume that a standard working surface as depicted in Fig. 2 exists. The conversion of kinetic to internal energy will then take place either in the forward or the reverse shock.

\subsubsection{Discrete ejections}

If the jets are composed of discrete ejections, then each ejection will eventually convert its kinetic energy into some form of internal energy via interaction with the ISM, not unlike the external shock encountered in GRB afterglows. During the phase of relativistic propagation of the ejection, either the forward or the reverse shock (or both) must be relativistic. It is inside of the relativistic portion of the shock that most of the energy is dissipated.

For a cold ballistically expanding ejection, most of the energy is dissipated in the forward shock. This is implied by the small opening angle $\theta$ of the ejection: as long as $\theta \sim c_{\mathrm{s}} / \Gamma_{\text {jet }} c \ll$ $1 / \Gamma_{\text {jet }}$ (with $\Gamma_{\text {jet }}$ being the bulk Lorentz factor of the ejection), the characteristic transverse size of the ejection $R$ is always much smaller than the distance $d$ over which it slows down, as seen in the frame of the blob: $R \sim \theta d \ll d / \Gamma_{\text {jet }}$ (here, the factor of $1 / \Gamma_{\text {jet }}$ accounts for the Lorentz contraction in going to the frame of the ejection). Thus, the deceleration time $d / \Gamma_{\text {jet }} c$ is much longer than the light crossing time of the ejection $R / c$, and the deceleration must occur in a sub-relativistic shock. This implies that the ejection is not heated to relativistic tempera- tures, while the forward shock must be relativistic, with shock velocity corresponding to $\Gamma_{\text {jet }}$.

The particles passing through this shock will have energies of order $\Gamma_{\text {jet }} m_{\mathrm{p}} c^{2} \leq \Gamma_{\text {jet }, 0} m_{\mathrm{p}} c^{2}$, where $\Gamma_{\text {jet }, 0}$ is the initial Lorentz factor of the ejection (before interaction with the ISM). Because the ejection is slowed down, particles of a spread in energies are created in the forward shock, though the spectrum will show a sharp turnover or cutoff at energies $e \gtrsim \Gamma_{\text {jet }, 0} m_{\mathrm{p}} c^{2}$ (below this cutoff, it is plausible that the spectrum rises with $\mathrm{d} N / \mathrm{d} \gamma \propto \gamma^{-3}$, see Appendix A).

\subsubsection{Continuous jets}

However, there are reasons to believe that at least in the well studied cases of GRS 1915+105 (Kaiser et al. 2000, though this question is up for debate) and in SS 433, as well as in the persistent radio outflows of low-hard state sources like GX 339-4 and Cyg X-1 the jets are continuous streams of matter. In which part of the shock the dissipation occurs in a continuous jet depends on the jet thrust and the external density: the advance speed of the jet is roughly given by ram pressure balance. The transition from forward to reverse shock dissipation then occurs when

$\frac{L}{A_{\text {shock }} \rho_{\mathrm{x}} c^{3}}=\frac{2 L_{38.5}}{l_{16}^{2} \theta_{0.1}^{2} n_{\mathrm{ISM}}} \sim 1$,

where $L_{38.5} \equiv L_{\text {kin }} / 10^{38.5} \mathrm{ergs} \mathrm{s}^{-1} \sim L_{\mathrm{kin}} / 3 \times 10^{38} \mathrm{ergs} \mathrm{s}^{-1}$ is the kinetic power, $l_{16}=l_{\text {jet }} / 10^{16} \mathrm{~cm}$ is the jet length, $A_{\text {shock }}$ the surface area of the shock, $\theta_{0.1} \equiv \theta / 0.1$ the jet opening angle in units of 0.1 radian or $5.7^{\circ}$, and $n_{\mathrm{ISM}} \equiv \rho_{\mathrm{x}} / m_{\mathrm{p}}$ the ISM particle density in units of $1 \mathrm{~cm}^{-3}$. Note that the working surface propagates, so $l_{16}$ is a measured quantity, given by the age of the source, its power, and the ISM density. Strictly speaking, the jet thrust will have to be measured in the shock frame, and the relativistic shock jump conditions applied, but in order to estimate which of the two shocks is going to be relativistic, the above approximation is sufficient.

The reverse shock is relativistic if the ratio on the left hand side of Eq. (2) is smaller than unity, the forward shock is relativistic if the ratio on the left hand side is larger than unity. For Galactic sources both cases can occur for appropriate external densities, depending on the length of the jet.

\subsection{Particle acceleration in relativistic shocks}

\subsubsection{Nucleon acceleration}

For non-relativistic shocks, it has long been known that multiple shock crossings can accelerate particles to ultra-relativistic energies. The most commonly accepted scheme is first order Fermi acceleration (e.g., Krymskii 1977; Blandford \& Ostriker 1978; Bell 1978; Blandford \& Eichler 1987), which typically produces a powerlaw distribution

$f(\gamma)=f_{0} \gamma^{-s}$

with canonical index $s \sim 2$.

For relativistic shocks the situation is not as clear cut. Several attempts have been made to solve the problem of particle acceleration at relativistic shocks, mostly in the limit of 
test particle acceleration. In general, it is found that powerlaw distributions with somewhat steeper spectra than in nonrelativistic shocks can be produced (Ellison \& Double 2002; Achterberg et al. 2001; Kirk \& Schneider 1987).

It is certain, however, that acceleration of particles in the shock must take place: Particles crossing the shock are by nature already relativistic in the downstream rest frame, with a typical Lorentz factor of $\Gamma_{\text {rel }}$, the relative Lorentz factor between upstream and downstream frames. The particle distribution leaving the shock is thus strongly anisotropic, and essentially mono-energetic. The randomization of this energy is then a question of the efficiency of the typical plasma processes often assumed to be present in populations of relativistic particles.

The simplest assumption is that the particle momenta are simply isotropized behind the shock. The shock acceleration kernel is then a delta function and a cold upstream plasma will be transformed into a narrow but relativistic energy distribution, the width $\sigma_{\Gamma}$ of which should roughly be given by the Lorentz transformed width $\sigma$ of the upstream energy distribution, $\sigma_{\Gamma} \sim \Gamma_{\text {rel }} \beta \sigma$, where $\Gamma_{\text {rel }}$ is the relative Lorentz factor between the upstream and downstream frames. The mean particle energy will be $\left\langle\gamma m c^{2}\right\rangle \sim \Gamma_{\text {rel }} m c^{2} \sim \Gamma_{\text {jet }} m c^{2}$.

If scattering by downstream turbulence or particle interactions is stronger, the particles can be thermalized, in which case a Maxwell-Boltzmann distribution according to the relativistic Rankine-Hugoniot jump conditions will be established. The main observational difference between these two cases will be the width of the energy distribution (see Fig. 5).

If a significant fraction of the particles can perform multiple shock crossings (which again hinges on effective scattering to return downstream particles to the shock), we expect a powerlaw-type distribution to be established. It is reasonable to assume that the first time escape fraction from the downstream region (i.e., the probability that a particle which crossed the shock only once) is of order $\mathcal{P}_{\text {esc }} \lesssim 90 \%$ (e.g., Achterberg et al. 2001), which implies that most of the particles will only cross the shock once (note that the escape probability in nonrelativistic shocks is generally very small for fast particles, though $\mathcal{P}_{\text {esc }}$ is of order unity for thermal particles, Bell 1978). The escape fraction in the upstream region is much smaller and generally neglected in calculations.

The particles re-crossing the shock will pick up another factor of order $\Gamma_{\text {rel }}^{2}$ in energy gain (Vietri 1995), which implies that a significant fraction of particles will be boosted to higher energies $\left(\gamma \geq \Gamma_{\text {rel }}^{3}\right)$. This fraction of the particles will contribute a significant amount of pressure to the post shock gas, which will modify the shock structure accordingly. Thus, the amount of energy accessible to the bulk of the particles which cross the shock only once is of the order $\left[1+\Gamma_{\text {rel }}^{2}\left(1 / \mathcal{P}_{\text {esc }}-1\right)\right]^{-1} \sim$ $50 \%-90 \%$.

The remaining fraction $1-\mathcal{P}_{\mathrm{esc}}$ of the particles will perform true Fermi acceleration. The low energy turnover of this distribution should be located roughly at $\gamma \sim \Gamma_{\text {rel }}^{3}$ (Vietri 1995) and subsequent shock crossings will produce features at energies of $\Gamma_{\text {rel }}^{2 i+1}$ (where $i$ enumerates the number of shock crossings). The similarity of this process to Compton upscattering was already mentioned in Sect. 2.3.
The superposition of features from multiple shock crossing cycles will lead to the formation of a powerlaw at high energies, very similar to the powerlaw produced by optically thin inverse Compton scattering (where the Lorentz transformations of the photon distribution to and from the particle rest frame are replaced by Lorentz transformations to and from the upstream fluid rest frame, assuming that scattering is strong enough to isotropize the particle distribution. The optical depth $\tau_{\text {IC }}$ is replaced by the return probability $1-\mathcal{P}_{\text {esc }}$.) The shock powerlaw slope $s_{\text {shock }}$ is determined by $\Gamma_{\text {rel }}$ and $\mathcal{P}_{\text {esc }}$ (e.g., Bell 1978):

$s_{\text {shock }} \sim 1-\frac{\ln \left(1-\mathcal{P}_{\mathrm{esc}}\right)}{\ln \left(\Gamma^{2}\right)}$

Thus, observationally, diffusive acceleration will lead to the presence of a second feature in the CR spectrum around $\sim \Gamma_{\text {jet }}^{3}$. This situation is shown in Fig. 5, where the powerlaw spectral component was plotted for $\Gamma_{\text {jet }}=5$. The energy of this broad peak coincides nicely with the peak in the excess CR proton component required in the HEMN model (Strong et al. 2000) to explain the $\gamma$-ray EGRET excess above $1 \mathrm{GeV}$.

We note that Achterberg et al. (2001) argue that in the absence of an efficient scattering mechanism, the average energy gain per particle will be restricted to a factor of order unity (rather than $\Gamma_{\text {rel }}^{2}$ ) for higher order shock crossings $(i \geq 2)$, and that the low energy turnover of the powerlaw component might thus be located at $\sim \Gamma_{\text {rel }}^{2}$ (rather than $\Gamma_{\text {rel }}^{3}$ ). The search for additional features in the CR spectrum at energies $\Gamma^{2}$ and $\Gamma^{3}$ might offer a potential way to test these predictions.

It is also possible that a population of relativistic protons already exists upstream of the shock. If the proton number density is equal to the electron number density, the bulk of the particles and of the energy will presumably be at the low energy end $(\gamma \sim 1)$, otherwise the estimates for kinetic energy flux in the jet would have to be increased accordingly, increasing the impact on the Galactic CR spectrum as well (by a factor of $\gamma_{0}$, the low energy cutoff of the distribution).

This population will be shifted to higher energies in the shock, and possibly experience further Fermi type acceleration. Thus, if a powerlaw of relativistic protons exists prior to the terminal shock with lower cutoff $\gamma_{0}$ and spectral index $s_{\text {jet }}$, the terminal shock should shift the lower cutoff roughly to $\Gamma_{\text {jet }} \gamma_{0}$, while the slope of the new powerlaw will be $\operatorname{Min}\left(s_{\text {jet }}, s_{\text {shock }}\right)$, where $s_{\text {shock }}$ is the powerlaw slope produced in the relativistic terminal shock.

While the particles produced in the shock must be relativistic at injection, the dynamical evolution of the shocked gas can reduce their energies significantly if adiabatic cooling is important before the particles can escape the shock region (radiative cooling of the nucleon distribution will be negligible). The diffusion of particles out of radio lobes and hot spots is a highly uncertain process and has not been studied in the necessary detail to answer this important question. Rather than discussing it at length, which would by far exceed the scope of this paper, we decided to include a short discussion in Appendix B, where we show that adiabatic losses do indeed pose a significant obstacle to particle escape (see also Fig. 5). 


\subsubsection{Electron acceleration}

CR protons do not emit any measurable amount of synchrotron radiation. Inverse Compton scattering on protons is also very inefficient. However, if diffusive acceleration is operating efficiently in the working surface, then the powerlaw electrons produced there should be detectable through their radiative signature. Tentative evidence of this powerlaw electron component are the radio lobes in the microquasars 1E 1740-294 and GRS 1758-258.

The spectral index of the non-thermal emission from the lobes of 1E 1740-294 is $\alpha \sim 0.7-0.9$ (Mirabel et al. 1992), corresponding to an electron spectrum of slope of $s \sim 2.4-2.8$. If the protons are injected at the working surface with the same slope (this would require that the electron spectrum is unaffected by radiative cooling and that the powerlaw electrons are not just advected through the shock from upstream), then the observed CR slope near the earth should be steepened by $\Delta s=1 / 2$ due to the energy dependence in the Galactic diffusion coefficient. This would yield a proton powerlaw slope of $s \sim 2.9-3.3$, somewhat steeper than the canonical CR slope of $s \sim 2.7$, though not significantly.

Part of the observed powerlaw electron distribution inside the tentative radio lobes of 1E 1740-294 and GRS 1758-258 could also have been advected from the jet. In fact, is is unclear whether electrons can be accelerated efficiently in a shock if protons are present, since the shock thickness will be set by the proton Larmor radius, which will be much larger than the electron Larmor radius (e.g., Achterberg et al. 2001). In this case, the electrons will not experience a shock at all, more likely, they will be accelerated adiabatically to a narrow component with energies of order $\Gamma_{\text {jet }} m_{\mathrm{e}} c^{2}$. Such a component will not be detectable through synchrotron radiation.

A detailed model of the energetic history of the particles in the lobes of 1E 1749-294 would be required to answer these questions.

\section{Observational consequences}

\subsection{Predicted contribution to the Galactic CR spectrum}

Based on the picture laid out in Sect. 3, we can try to predict what might be observed in the CR spectrum due to the presence of Galactic microquasars.

First, it is obvious that close enough to a powerful relativistic jet source the locally observed CR spectrum will be completely dominated by the CRs produced in the terminal shock of the jet. However, it is clear that the powerlaw spectrum observed near earth is not dominated by a narrow component of microquasar origin - the current spectral limits rule out any contribution greater than a few percent.

In a simple isotropic diffusion picture, the CR energy density in the environment of a continuously active source will fall roughly like the inverse distance to the source $r^{-1}$ (see Eq. (8)) for large $r$ much larger than a particle mean free path, $r \gg \kappa / c \sim 0.2 \mathrm{pc} H_{\mathrm{kpc}}^{2} / \tau_{15}$, and smaller than the Galactic disk height, $r \lesssim H_{\text {disk }}=1 \mathrm{kpc} H_{\mathrm{kpc}}$.
Given the observed CR energy density of

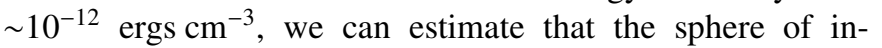
fluence of a given source, defined as the region inside which the source contributes more than $30 \%$ of the total measured CR power (at which level it would enter the realm of detectability of by $A M S 02$ ) has a radius of order

$R_{30 \%} \sim 1 \mathrm{kpc} \frac{L_{38.5}}{\kappa_{28.3}}$

The time it takes the source to populate this sphere with CRs is roughly

$\tau_{30 \%} \sim 15 \times 10^{6} \mathrm{yrs} \frac{L_{38.5}^{2}}{\kappa_{28.3}^{3}}$.

Particles that reach the edge of the Galactic disk will be siphoned off into the Galactic halo. Thus the CR flux will diminish rapidly, roughly exponentially, beyond a source distance of order the disc thickness, $r>H$. It would take source activity longer than $10^{8}$ yrs to fill the Galactic halo and the disk.

The well known microquasars mentioned above are all located much further from the solar system than this limit. However, if a source similar to, say, GRS $1915+105$ had been active in the solar neighborhood (inside about $1 \mathrm{kpc}$ ) within the last $\sim 10^{7} \mathrm{yrs}$, our local CR flux should show a clear sign of the contribution from this source.

In this context it is important to mention that GRO J165540, V4641 Sgr, Cyg X-3 (and also SS433) are known to be in high-mass X-ray binaries. Their lifetimes are therefore expected to be short. If such a relativistic jet black hole binary was located in the Orion nebula region within the past $10^{6} \mathrm{yrs}$, we should be able to detect a strong signal in the low energy CR spectrum from this source alone.

Far enough away from any single source, an observer will measure the time averaged contribution from all Galactic sources, washed out by CR diffusion (similar to the situation described in Strong \& Moskalenko 2001). Since sources will likely follow a distribution of Lorentz factors of width $\Delta \Gamma_{\text {jet }}$, the observed signal will be smeared out over at least that width. Any intrinsic width of the produced CR spectrum will add to this effect, as well as broadening effects like solar modulation and scattering off of interstellar turbulence.

In Fig. 7 we have plotted possible contributions to the CR proton spectrum from a single Galactic jet source. Depending on how much we have underestimated the power in Galactic jets and how much adiabatic losses of particles trapped in adiabatically expanding shock will suffer, we might over or underestimate the contribution. Taking the figure at face value, however, it seems likely that a contribution at the few percent level can be expected in the energy region of a few $\mathrm{GeV}$.

\subsection{Detectability of narrow features in the CR spectrum}

Detecting and positively identifying such a CR component will be a formidable challenge. The advent of the high sensitivity AMS 02 instrument and of the solar minimum might make it possible, however. Given the preliminary specifications of 


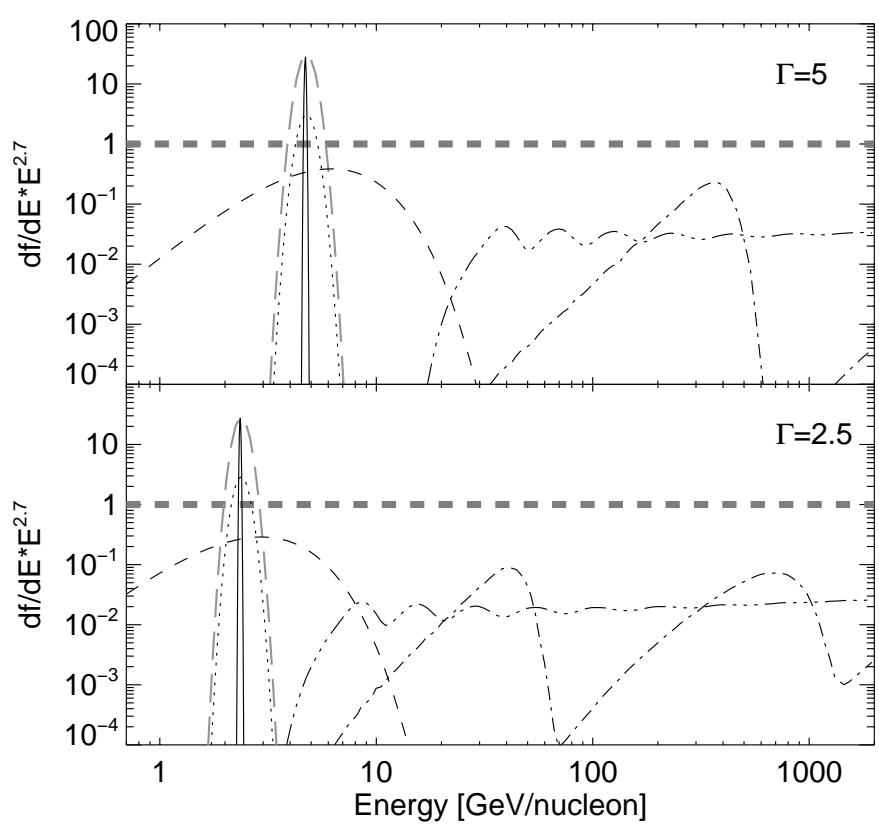

Fig. 7. Toy model of the microquasar contribution to the $\mathrm{CR}$ spectrum, for a single microquasar situated in a low mass X-ray binary, active for $\tau \gtrsim 1.5 \times 10^{7} \mathrm{yrs}$ on the level of $3 \times 10^{38} \mathrm{ergs} \mathrm{s}^{-1}$ (similar to GRS $1915+105$ ), and at a distance of $1 \mathrm{kpc}$. For simplicity, we assumed the source was operating with uniform bulk Lorentz factor $\Gamma_{\text {jet }}=5$ (top) and $\Gamma_{\text {jet }}=2.5$ (bottom). The curves are normalized relative to the measured differential Galactic CR background spectrum (thick grey dashed line). Shown are the same curves as in Fig. 5: narrow feature for upstream temperature of $T \sim 7 \times 10^{10} \mathrm{~K}$ (dotted line) and for $T \sim 7 \times 10^{8} \mathrm{~K}$ (solid line), Maxwellian with $k T \sim \Gamma_{\text {jet }} m_{\mathrm{p}} c^{2} / 3$ (dashed line), multiply scattered component for efficient pitch angle scattering (dash-dotted line) and for inefficient pitch angle scattering (dash-triple-dotted line), and the relative contribution of heavy elements for metallicity 10 times the solar value (long-dashed grey curve), as seen in GRO J1655-40 and V4641 Sgr. Each spectral component has been steepened by $E^{-1 / 2}$ to account for the energy dependence of the diffusion coefficient.

AMS 02, we can estimate the detectability of spectral features such as produced by microquasars. The rigidity resolution (i.e., energy resolution) of the instrument is expected to be around $2 \%$ in the crucial range from 1 to $10 \mathrm{GeV}$, which will easily be sufficient to identify and resolve even the narrowest feature in Fig. 7.

For an effective area of order $0.4 \mathrm{~m}^{2} \mathrm{sr}$, the expected total $\mathrm{CR}$ proton count rate by $A M S 02$ in the energy range from 1 to $10 \mathrm{GeV}$ should be of the order of $10^{3} \mathrm{~s}^{-1}$. At $2 \%$ energy resolution, this implies a detection rate of about $2 \times 10^{8} \mathrm{yr}^{-1} \mathrm{bin}^{-1}$, with a relative Poisson-noise level of order $10^{-4}$. Calibration and other systematic errors will likely dominate the statistics, however, these numbers are encouraging, and we expect that a source at the few-percent level will be detectable with AMS 02 .

The heavy element sensitivity of AMS 02 will share similar characteristics: for the same energy resolution and effective area, the detection rates of carbon and iron, for example, should be of order $4 \times 10^{5} \mathrm{~s}^{-1} \mathrm{bin}^{-1}$ and $4 \times 10^{4} \mathrm{~s}^{-1} \mathrm{bin}^{-1}$ respectively. Aside from AMS 02, signatures might be detected by other instruments, and even existing data sets might contain signals. Identification would require scanning these data with

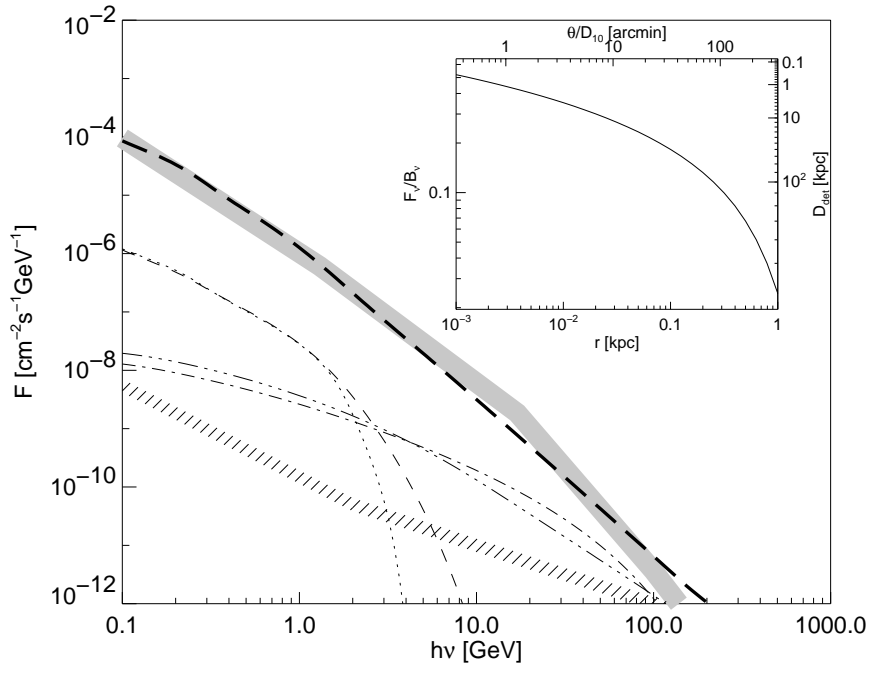

Fig. 8. Toy model for the gamma ray signature produced in a microquasar CR halo via pion decay (including $\pi^{0}$ decay and bremsstrahlung from secondary electrons and positrons). Curves were calculated assuming a source active for over $\tau \gtrsim 15 \times 10^{6} \mathrm{yrs}$ with an average power of $L_{\mathrm{kin}}=3 \times 10^{38} \mathrm{ergs} \mathrm{s}^{-1}$, at a distance of $10 \mathrm{kpc}$, and for an ISM particle density of $1 \mathrm{~cm}^{-3}$, assuming CRs are lost once they have reached the edge of the Galactic disk at about $1 \mathrm{kpc}$ distance from the source. Labes according to Fig. 5. Dotted line: Gamma ray signature from narrow component of the microquasar halo; dashed line: from thermalized component; dash-dotted line: from "powerlaw type component" in the case of efficient scattering; dash-triple-dotted line: from "powerlaw type component" in the case of inefficient scattering. Thick grey line: EGRET diffuse gamma ray background at the position of GRS 1915+105 (Hunter et al. 1997), assuming the same solid angle as subtended by the source. Thick long dashed line: background gamma ray emission over the same solid angle modeled assuming the proton CR spectrum measured near earth and an average ISM density of $1 \mathrm{~cm}^{-3}$. Hatched line: GLAST sensitivity. Models were computed using the GALPROP routines by Moskalenko \& Strong (1998). Insert: Angular dependence of the source contribution to the gamma ray flux $F_{v}$, relative to the background flux $B_{v}$ at a photon energy of $1 \mathrm{GeV}$ (see Eq. (8)). The right Y-axis shows the source distance at which the GLAST sensitivity is reached for the value of $F_{v} / B_{v}$ shown in the curve, the top X-axis shows the angle $\theta=r / D$ in units of $\operatorname{arcmin} / D_{10}$ where $D_{10}$ is the source distance in units of $10 \mathrm{kpc}$.

high spectral resolution. Note that the effects of solar modulation will broaden any narrow spectral component significantly. Results by Labrador \& Mewaldt (1997) demonstrate that a line at $\sim 5 \mathrm{GeV}$ will be broadened by $\sim 1 \mathrm{GeV}$, (less at higher energies) though this effect will be reduced at solar minimum.

\subsection{Gamma-ray emission from pion decay}

As the CRs produced in microquasars travel traverse the Galaxy, they will encounter the cold ISM. The interaction of a CR proton (by far the most abundant and thus most energetic component of the CR spectrum) with a cold ISM proton can lead to secondary particle production and to the emission of gamma rays via several channels, the most important of which is $\pi^{0}$ decay. 
Using the toy model presented in Fig. 7, we can estimate how much gamma ray flux can be expected from the CR halo of a powerful microquasar and compare it to the background flux from the Galaxy. We assume that the CRs diffuse away from the source until they reach the Galactic halo, approximated as a zero pressure boundary condition at radius $R \sim H_{\text {disk }}=1 \mathrm{kpc} H_{\mathrm{kps}}$ (assuming spherical symmetry for simplicity). The result is shown in Fig. 8.

Note that the gamma ray signal even for a source of average kinetic power of $L_{\text {kin }}=3 \times 10^{38} \mathrm{ergs} \mathrm{s}^{-1}$ is small compared to the background signal coming from the same solid angle $\left(\pi R^{2}\right)$. However, because the CR density increases towards the center of the source, higher spatial resolution can improve the signalto-noise ratio somewhat. For a spherically symmetric cloud of CRs with luminosity $L$ and vanishing pressure at the boundary $R \sim H_{\text {disk }}$, the density follows

$$
\begin{aligned}
& n_{\mathrm{CR}}(r, \gamma)=\frac{f(\gamma)}{\int \mathrm{d} \gamma^{\prime} \gamma^{\prime} m c^{2} f\left(\gamma^{\prime}\right)} \frac{L}{4 \pi \kappa}\left(\frac{1}{r}-\frac{1}{R}\right) \\
& =6 \times 10^{-11} \mathrm{~cm}^{-3} \frac{f(\gamma)}{\int \mathrm{d} \gamma^{\prime} \gamma^{\prime} m c^{2} f\left(\gamma^{\prime}\right)} \frac{L_{38.5} \tau_{15}}{H_{\mathrm{kpc}}^{2} R_{\mathrm{kpc}}}\left(\frac{R}{r}-1\right) .
\end{aligned}
$$

Obviously, then, the gamma ray surface brightness of such a source is centrally peaked and thus the best observing strategy is to try to resolve the source. The photon flux $F_{v}$ from within an angular distance $\theta \leq r / D$ from the source position (here, $D$ is the physical source distance, while $\theta$ the on-sky angular distance from the source position) is then proportional to

$$
\begin{aligned}
F_{v}\left(\theta \leq \frac{r}{D}\right) \sim & \frac{n_{\mathrm{ISM}} c}{4 \pi D^{2}} \frac{L R^{2}}{3 \kappa}\left(\frac{\int \mathrm{d} \gamma^{\prime} \sigma_{v}\left(\gamma^{\prime}\right) f\left(\gamma^{\prime}\right)}{\int \mathrm{d} \gamma^{\prime} \gamma^{\prime} m c^{2} f\left(\gamma^{\prime}\right)}\right) \\
\times & \left\{1+3 r^{2}\left[\ln \left(1+\sqrt{1-r^{2}}\right)-\ln (r)\right]\right. \\
& \left.-\left(1+r^{2}\right) \sqrt{1-r^{2}}\right\}
\end{aligned}
$$

whereas the galactic background flux $F_{v \text {, back }}$ for the same solid angle in the small angle approximation is simply proportional to $(r / D)^{2}$. Thus, the source contribution relative to the background, measured as $F_{v \text {, source }} / F_{v \text { back }}$, rises towards lower $r$, which we have plotted in the insert in Fig. 8.

\subsubsection{Sub-cosmic rays from sub-relativistic sources like SS433}

Objects like SS433 might be important sources of sub-cosmic rays in the Galaxy: the cold thermal ions carried in the jet at $0.26 c$ will be accelerated to energies of order $E_{\text {ion }} \equiv E_{30} \times$ $30 \mathrm{MeV} /$ nucleon. These particles will suffer severe ionization and Coulomb losses which will prevent them from traveling further than about $250 \mathrm{pc}$ from the source.

However, they could act as a significant ionization source for the surrounding medium: the ionization loss timescale for a particle with energy $E=30 \mathrm{MeV} E_{30}$ is of order (Ginzburg 1979)

$\tau_{\text {ion }} \sim 2 \times 10^{6}$ yrs $\frac{E_{30}^{3 / 2}}{n_{\text {ISM }}}$,

much shorter than the expected lifetime of the particles inside the Galactic disk, i.e., the particles will deposit their energy in the vicinity of the source, especially if the source is located within a molecular cloud.

Furthermore, the excitation of nuclear $\gamma$-ray emission lines by interaction of these sub-cosmic rays with interstellar heavy ions of $\mathrm{C}, \mathrm{O}, \mathrm{Fe}$, and other elements might be detectable by INTEGRAL.

\subsection{Cold electron-positron jets}

If the jet consists chiefly of relatively cold electron-positron plasma, and if dissipation occurs mostly in the reverse shock, then the jet terminus will produce relativistic electrons and positrons with energies of the order of $\Gamma_{\text {jet }} m_{\mathrm{e}} c^{2} \sim 2.5 \mathrm{MeV}$, which will then begin to diffuse into the ISM. Such positrons and electrons could produce additional bremsstrahlung radiation at energies of a few hundreds of keVs up to $2.5 \mathrm{MeV}$. Much like mildly relativistic protons, these electrons will contribute to the heating of the ISM due to the ionization losses, but much more important for future observations might be the electronpositron annihilation line at $511 \mathrm{keV}$.

For an integrated mechanical luminosity of $L_{\text {kin }}=3 \times$ $10^{38} \mathrm{ergs} \mathrm{s}^{-1} L_{38.5}$ of the entire ensemble of Galactic relativistic jets, the flux of positrons carried by jets is

$\dot{N}_{\mathrm{e}^{+}}=\frac{L_{\mathrm{kin}}}{2\langle\gamma\rangle \Gamma_{\text {jet }} m_{\mathrm{e}} c^{2}} \sim 4 \times 10^{43} \mathrm{~s}^{-1} \frac{L_{38.5}}{\Gamma_{5}\langle\gamma\rangle}$,

where $\langle\gamma\rangle$ is the mean random Lorentz factor of the electrons/positrons in the jet plasma. Note that this estimate will be independent of whether dissipation occurs mostly in the forward or reverse shock. Assuming that these positrons can diffuse out of the radio plasma they are originally confined in, the estimated luminosity of the annihilation line will be of the order of $L_{\text {ann }} \sim L_{\text {jet }} /\langle\gamma\rangle$.

This is actually comparable to the total amount of positrons annihilating in the Galaxy according to the observations of the $\mathrm{e}^{+} / \mathrm{e}^{-}$annihilation line from $O S S E / G R O, \dot{N}_{\mathrm{e}^{+}, \mathrm{Gal}} \sim 3 \times 10^{43} \mathrm{~s}^{-1}$ (Purcell et al. 1997). If Galactic jets are in fact composed of electron-positron plasma, this measurement immediately implies one of the following conclusions: a) either the mechanical luminosity of these jets is not far above our relatively conservative estimate of $3 \times 10^{38} \mathrm{ergs} \mathrm{s}^{-1}$, or b) the pair plasma is not cold, i.e., $\langle\gamma\rangle \gg 1$, or c) diffusion of particles across the magnetic boundary of the remnant jet plasma is very inefficient, in which case many Galactic "radio relics" should exist, not unlike in the case of radio relics from radio loud AGNs in the intracluster medium (e.g., Ensslin et al. 1998).

The Integral SPI spectrometer and the IBIS imager would be able to measure the increase in the annihilation line flux towards microquasars located away from the Galactic center (where the background is highest) like GRS1915+105, and to measure the line width if it could be detected. These measurements could be very helpful in constraining the particle content of relativistic Galactic jets.

\section{Conclusion}

We have argued that Galactic microquasars produce cosmic rays with energies of a few $\mathrm{GeV} /$ nucleon. Under preferable 
conditions (i.e., if particles in the jet are cold and do not suffer significant adiabatic losses before escaping the acceleration region) this component should be detectable as a narrow peak with energy $\sim \Gamma_{\text {jet }} m_{\mathrm{p}} c^{2} /$ nucleon in the Galactic CR spectrum. The superposition of several such peaks would then reflect the distribution of Lorentz factors of Galactic jet sources.

In addition, diffusive acceleration of particles might produce a powerlaw distribution of particles with a low energy turnover at energies around $\Gamma_{\mathrm{jet}}^{2} m_{\mathrm{p}} c^{2}$ to $\Gamma_{\text {jet }}^{3} m_{\mathrm{p}} c^{2}$, visible as an edge-like feature in the CR spectrum.

The locally measured contribution to the CR spectrum will be strongly dominated by sources operating close by (within a distance of about one Galactic disk height and within the past $10^{7} \mathrm{yrs}$ ), since at distances much larger than that the contribution from a given microquasar falls off exponentially. We estimate the global energy content in the CR component accelerated in Galactic relativistic jets to be at the $0.1 \%$ to $10 \%$ level of the total Galactic CR luminosity.

This CR contribution from Galactic relativistic jet sources might be strongly overabundant in heavy elements, reflecting the composition of the accretion disk where the jets are launched. Thus, it is possible that the chemical abundance measured in the $\mathrm{GeV}$ region (where we expect the contribution from jets to show the strongest effect) will differ slightly from the chemical composition at higher energies.

While signatures of the CR component from microquasars might already be buried in existing data, the upcoming solar minimum and the launch of $A M S O 2$ will offer ideal conditions to search for this component and to put constraints on the microquasar activity in the nearby universe.

We suggested that the absence of any observable traces of microquasar component in the cosmic ray proton spectrum could be used to argue in favor of electron-positron jets. For this case, we showed that existing OSSE/GRO observations of the Galactic electron-positron annihilation rate can be used to limit the power in cold electron-positron jets inside the Galaxy to $L_{ \pm}<3 \times 10^{38} \mathrm{ergs} \mathrm{s}^{-1}$.

Acknowledgements. We would like to thank Roger Blandford, Andrei Bykov, Richard Mewaldt, Igor Moskalenko, and Vladimir Ptuskin for insightful discussions and comments on the manuscript. We would also like to thank Andrew Strong for providing access to the GALPROP code. We would like to thank the referee Rob Fender for helpful comments regarding all aspects of the paper. Rashid Sunyaev, as a Gordon Moore Scholar, thanks Caltech for its hospitality during the work on this paper. This research has made use of the public GBI monitoring database hosted by NRAO.

\section{Appendix A: The particle spectrum produced in decelerating ejections}

It is straight forward to calculate the particle distribution produced by single pass shock acceleration in a decelerating ejection in the ultra-relativistic limit, assuming that one has knowledge of the single pass shock acceleration kernel at a given shock velocity $\Gamma_{\text {rel }}$.
Take an ejection of initial mass $M_{0}$ and Lorentz factor $\Gamma_{0}$, which is sweeping up and shocking external matter. The total energy of the ejection and the swept up matter is

$E \sim \Gamma c^{2}\left(M_{0}+\int_{0}^{N} \mathrm{~d} N^{\prime} \Gamma\left(N^{\prime}\right) m_{\mathrm{p}}\right)$

where the integration is over the number $N$ of swept up particles with mass $m_{\mathrm{p}}$ at a given ejection Lorentz factor $\Gamma$, and the extra factor of $\Gamma$ inside the integral takes account of the shock acceleration of the swept up particles.

Since $E$ is conserved, we can take the derivative of Eq. (A.1) with respect to $\Gamma$, and arrive at

$\frac{\mathrm{d} N}{\mathrm{~d} \Gamma} \sim \frac{\left(\int_{0}^{N} \mathrm{~d} N^{\prime} \Gamma\left(N^{\prime}\right) m_{\mathrm{p}}+M_{0}\right)}{\Gamma^{2} c^{2}} \sim \frac{E}{m_{\mathrm{p}} c^{2}} \Gamma^{-3}$

for $1 \ll \Gamma \leq \Gamma_{0}$. For $\Gamma \sim 1$, the non-relativistic corrections reduce the amount of energy available, leading to a low energy cutoff at $\Gamma \sim 1$ and a high energy cutoff at $\Gamma_{0}$.

To arrive at the observed particle distribution $\mathrm{d} N / \mathrm{d} \gamma$, this must be convolved with the single shock acceleration kernel $\gamma(\Gamma)$, however, for a narrow kernel, such as assumed in this paper, the powerlaw approximation seems sufficient: $\mathrm{d} N / \mathrm{d} \gamma \propto \gamma^{-3}$.

\section{Appendix B: Cosmic ray diffusion and adiabatic cooling}

An important question is whether the particles produced in the shock discussed in Sect. 3.3.1 can indeed diffuse out of the shock region, in which case they will freely escape and propagate through the Galaxy essentially with the energy obtained in the shock, or whether they are trapped inside the shocked gas until it expands adiabatically after the shock has passed and activity has ceased. In the latter case, the particles will lose a significant amount of energy to adiabatic expansion.

Following the discussion in Sect. 3.3.1, we distinguish two cases: dissipation in the forward and in the reverse shock.

The escape time of the particles out of the shock can be estimated as

$\tau \sim \frac{R_{\text {shock }}^{2}}{\kappa}$

where $R_{\text {shock }}$ is the typical size scale of the shock and $\kappa$ the relevant diffusion coefficient.

While the shocked ISM must still be magnetically connected with the unshocked ISM, the jet plasma will be situated on field lines advected out from the central engine, which are likely not connected with the ISM. In the forward shock, the relevant diffusion coefficient should then be taken as $\kappa_{\text {forward }} \sim \kappa_{\|}$, the diffusion coefficient parallel to the mean magnetic field, while for the reverse shock one has to consider diffusion across the magnetic boundary of the contact discontinuity between shocked jet plasma and shocked ISM in addition to diffusion to the contact discontinuity and away from it.

A lower limit on the diffusion time out of the shock is thus given by the value for the forward shock, since the particles which have diffused out of the reverse shock must, in addition, also propagate through the forward shock. 


\section{B.1. Forward shock}

Using the simple approximate expression for $\kappa_{\|}$(e.g., Kennel \& Petschek 1966), and assuming a Kolmogorov turbulence spectrum for the magnetic field originating on scales of order the shock size $R_{\text {shock }}=l_{\text {jet }} \theta=10^{15} \mathrm{~cm} l_{16} \theta_{0.1}$ and containing a fraction $\epsilon \equiv B_{\text {turb }}^{2} / B_{\text {tot }}^{2}$ of the total magnetic energy, the parallel diffusion coefficient for a particle with energy $\gamma m c^{2} \sim \Gamma_{\text {jet }} m c^{2}$ can be written as

$$
\begin{aligned}
\kappa_{\|} & \sim \frac{2}{\pi} v r_{\mathrm{G}} \frac{v_{\text {gyro }}}{v_{\text {scatter }}} \sim \frac{2 r_{\mathrm{G}} c}{\pi} \frac{B^{2}}{3 k B_{k}^{2}} \\
& \sim \frac{2 r_{\mathrm{G}} c}{3 \pi \epsilon}\left(\frac{R_{\text {shock }}}{r_{\mathrm{G}}}\right)^{2 / 3} \sim \frac{2 c}{3 \pi \epsilon}\left(\frac{\gamma m c^{2}}{e B} l_{\text {jet }}^{2} \theta^{2}\right)^{1 / 3} .
\end{aligned}
$$

Here, $v_{\text {scatter }}$ is the scattering frequency for particles with gyro radius $r_{\mathrm{G}}$ off magnetic turbulence with energy density $k B_{\mathrm{k}}^{2} / 8 \pi$ and wave numbers of order $k \sim r_{\mathrm{G}}^{-1}$. Scattering can also be induced by collective resonant interactions of particles with the field, in which case the parameter $\epsilon$ denotes the efficiency of this process.

Writing the shock area as $A_{\text {shock }}=\pi R_{\text {shock }}^{2}=\pi \times$ $10^{30} \mathrm{~cm}^{2} \theta_{0.1}^{2} l_{16}^{2}$ gives an approximate hot spot pressure $p_{\text {shock }}$ of

$p_{\text {shock }} \sim \frac{L_{\text {kin }}}{A_{\text {shock }} c}=3 \times 10^{-3} \operatorname{ergs~cm}^{-3} L_{38.5} l_{16}^{-2} \theta_{0.1}^{-2}$.

If the magnetic field is at a fraction $\varphi$ of the equipartition field, $\varphi \equiv B / B_{\text {eq }}$, we have $B \equiv \varphi B_{\text {eq }} \sim 0.5 \mathrm{G} \varphi L_{38.5}^{1 / 2} / l_{16} \theta_{0.1}$.

The comoving (i.e., measured in the frame of the shocked plasma) limit to the proton escape time $\tau_{\|}^{\prime}$ is then

$$
\begin{aligned}
\tau_{\|}^{\prime} \gtrsim \frac{R_{\text {shock }}^{2}}{\kappa_{\|}} & \sim l_{\text {jet }} \theta \frac{3 \pi \epsilon \varphi^{1 / 3}}{2 c}\left(\frac{e}{\gamma m c^{2}}\right)^{1 / 3}\left(\frac{L_{\text {jet }}}{\pi c}\right)^{1 / 6} \\
& \sim 4 \times 10^{7} \mathrm{~s} \frac{l_{16} \theta_{0.1} \epsilon \varphi^{1 / 3} L_{38.5}^{1 / 6}}{\Gamma_{\text {jet }}^{1 / 3}}
\end{aligned}
$$

If we write the Lorentz factor of the shocked ISM gas as $\Gamma^{\prime}$, then time dilation gives $\tau_{\|}=\Gamma^{\prime} \tau_{\|}^{\prime}$ in the observer's frame.

If the turbulent velocity inside the region of interest is comparable to the expansion velocity, and if large scale turbulence is present (which was the underlying assumption in out estimate of $\epsilon$ above), then turbulent transport could aid particle escape: in a simple mixing length approach, the diffusion coefficient can be approximated by $\kappa_{\text {turb }} \sim 1 / 3 v_{\text {turb }} l_{\text {turb }}$, where $v_{\text {turb }}$ is the characteristic turbulent velocity and $l_{\text {turb }}$ the scale length of the largest scale turbulence. The turbulent transport time is then

$\tau_{\text {turb }} \sim \frac{3 R_{\text {shock }}^{2}}{v_{\text {turb }} l_{\text {turb }}} \sim 10^{7} \mathrm{~s} \frac{\dot{R}_{\text {shock }}}{v_{\text {turb }}} \frac{0.1 R_{\text {shock }}}{l_{\text {turb }}}$.

If the shock region is expanding roughly ballistically as it propagates, the adiabatic loss time scale measured in the observer's frame is

$\tau_{\mathrm{ad}}=\frac{\gamma}{\dot{\gamma}}=4 \frac{p}{\dot{p}}=\frac{R_{\text {shock }}}{\dot{R}_{\text {shock }}} \sim 3 \times 10^{5} \mathrm{~s} l_{16}\left(\frac{c}{\dot{l}}\right)$.

If the forward shock is relativistic, we need to take time dilation into account when comparing $\tau_{\text {ad }}$ and $\tau_{\|}$. It follows that the adiabatic loss time scale $\tau_{\mathrm{ad}}$ is longer than the escape time only if

$\epsilon \varphi L_{38.5}^{1 / 6} \theta_{0.1} \frac{\dot{l}_{\text {jet }}}{c} \Gamma_{5}^{2 / 3}<4 \times 10^{-3}$.

Since both $\epsilon$ and $\varphi$ might be significantly smaller than $10^{-5}$, it is not implausible that $\tau_{\|} \lesssim \tau_{\text {ad }}$. In the case of strong turbulent transport, this condition simplifies to

$v_{\text {turb }} l_{\text {turb }}>3 R_{\text {shock }} \dot{R}_{\text {shock }}$.

If the external pressure is parameterized as $p_{\text {ISM }} \equiv$ $10^{-11} \mathrm{ergs} \mathrm{cm}^{-3} p_{-11}$, the maximum reduction in particle energy possible through adiabatic losses is roughly

$$
\begin{aligned}
\left(\frac{p_{\text {ISM }}}{p_{\text {shock }}}\right)^{1 / 4} & \sim 10^{-2}\left(\frac{p_{-11} l_{16}^{2} \theta_{0.1}^{2}}{L_{38.5}}\right)^{1 / 4} \\
\lesssim \frac{\langle\gamma-1\rangle_{\text {min }}}{\langle\gamma-1\rangle_{\text {shock }}} & <\left(\frac{p_{\text {ISM }}}{p_{\mathrm{s}}}\right)^{2 / 5} \\
& \sim 6 \times 10^{-4}\left(\frac{p_{-11} l_{16}^{2} \theta_{0.1}^{2}}{L_{38.5}}\right)^{2 / 5},
\end{aligned}
$$

where the left hand side of the inequality corresponds to a relativistic equation of state in the hot spot and the right hand side to a non-relativistic equation of state. This, of course, means that a large fraction of the energy injected originally into CRs could be lost adiabatically, reducing the amount of energy available (estimated in Sect. 2.1) by up to a factor of $\sim 1000$.

\section{B.2. Reverse shock}

In jets where dissipation occurs mainly in the reverse shock, the jet geometry is similar to AGN jets (i.e., the jets are effectively reflected by the ISM, thus inflating a cocoon with spent jet fuel).

Particles accelerated in the shock will eventually be advected out of the shock region and into the cocoon (see Fig. 2). The timescale for this process is

$\tau_{\text {advect }} \sim \frac{R_{\text {shock }}}{v_{\text {advect }}} \sim 3 \times 10^{4} \mathrm{~s} l_{16} \theta_{0.1}\left(\frac{c}{v_{\text {advect }}}\right)$,

where $v_{\text {advect }} \lesssim c / 3$, since the limiting downstream velocity in a strong relativistic shock is $c / 3$.

The diffusion time towards the contact discontinuity is given by Eq. (B.4). In order to enter the forward shock, the particules have to propagate across the magnetic boundary at the contact discontinuity, which introduces an additional crossfield diffusion term. The contact discontinuity will have a typical thickness of the order of the Larmor radius $r_{\mathrm{G}}$ of the particles, thus the particles will have to traverse a region of size $r_{\mathrm{G}}$ perpendicular to the field in order to cross the contact discontinuity (this approximation is valid as long as the parallel diffusion time over one coherence length of the field is longer than the perpendicular diffusion time across one Larmor radius, otherwise the perpendicular diffusion time across one coherence length should be used). The lower limit to the diffusion time across the field is then $\tau_{\perp} \gtrsim \frac{r_{\mathrm{G}}^{2}}{\kappa_{\perp}}$. 
The perpendicular diffusion coefficient $\kappa_{\perp}$ can be approximated as $\kappa_{\|}\left(k B_{\mathrm{k}}^{2} / B^{2}\right)^{2}$ (e.g., Parker 1965), i.e.,

$$
\begin{aligned}
\tau_{\perp} & \sim \tau_{\|}\left(\frac{r_{\mathrm{G}}}{R_{\text {shock }}}\right)^{2}\left(\frac{\kappa_{\|}}{\kappa_{\perp}}\right) \sim \tau_{\|}\left(\frac{r_{\mathrm{G}}}{R_{\text {shock }}}\right)^{2}\left(\frac{B^{2}}{k B_{k}^{2}}\right)^{2} \\
& \sim \frac{3 \pi}{2 c \epsilon} R_{\mathrm{s}}^{2 / 3} r_{\mathrm{G}}^{1 / 3} \sim 160 \mathrm{~s} \frac{\gamma^{1 / 3} l_{16} \theta_{0.1}}{\epsilon L_{38.5}^{1 / 6} \varphi^{1 / 3}} .
\end{aligned}
$$

The total diffusion time out of the reverse shock should then be of the order of $\tau \sim \tau_{\|}+\tau_{\perp}$, which has a minimum when $\left(\epsilon \varphi^{1 / 3} L_{38.5}^{1 / 6} / \Gamma_{\text {jet }}^{1 / 3}\right)_{\min } \sim 3 \times 10^{-3}$, with $\tau_{\min } \sim 2 \times 10^{5} l_{16} \theta_{0.1}$. Thus, even in the optimal case, the diffusion time out of the reverse shock is longer than the advection time unless $v_{\text {advect }} \lesssim$ $0.1 c$.

Thus, it is rather likely that the bulk of the particles are advected out of the shock and into the cocoon. The pressure driven expansion of a cocoon can be approximated by a simple spherically symmetric model: Dimensional analysis suggests that the size of the cocoon $R_{\mathrm{c}}$ follows a simple scaling (Castor et al. 1975)

$R_{\mathrm{c}} \sim\left(\frac{L t^{3}}{\rho_{\mathrm{ISM}}}\right)^{1 / 5}$.

Using this scaling to obtain order of magnitude estimates of the conditions inside the cocoon, we can write the cocoon pressure $p_{\mathrm{c}}$ as

$$
\begin{aligned}
p_{\mathrm{c}} & \sim \frac{1}{2} \rho_{\mathrm{ISM}} \dot{R}_{\mathrm{c}}^{2} \\
& \sim 2 \times 10^{-5} \mathrm{ergs} \mathrm{cm}^{-3} R_{16}^{-4 / 3} L_{38.5}^{2 / 3} n_{\mathrm{ISM}}^{1 / 3},
\end{aligned}
$$

where $R_{16}=R_{\mathrm{c}} / 10^{16} \mathrm{~cm} \sim l_{16}$ is the cocoon radius. The magnetic field is

$B_{\mathrm{c}} \sim 3 \times 10^{-2} \mathrm{G} \varphi R_{16}^{-2 / 3} L_{38.5}^{1 / 3} n_{\mathrm{ISM}}^{1 / 6}$.

Comparison of Eq. (B.13) with Eq. (B.3) shows that a (relativistic) particle advected out of the shock into the cocoon will suffer adiabatic losses of the order

$$
\frac{\langle\gamma-1\rangle_{\mathrm{c}}}{\langle\gamma-1\rangle_{\text {shock }}} \sim\left(\frac{p_{\mathrm{c}}}{p_{\mathrm{s}}}\right)^{1 / 4} \sim 0.3\left(\frac{\theta_{0.1}^{2} R_{16}^{2 / 3} n_{\mathrm{ISM}}^{1 / 3}}{L_{38.5}^{1 / 3}}\right)^{1 / 4}
$$

which is only weakly dependent on the source parameters.

Once the particles are inside the cocoon, the escape time is again given by $\tau \sim \tau_{\|}+\tau_{\perp}$ with the expressions for $\tau_{\|}$and $\tau_{\perp}$ from Eqs. (B.4) and (B.11), though with different values:

$\tau_{\|} \sim 7 \times 10^{8} \mathrm{~s} R_{16}\left[\epsilon \varphi^{1 / 3} R_{16}^{1 / 9} \Gamma^{-1 / 3} L_{38.5}^{1 / 9} n_{\mathrm{ISM}}^{1 / 18}\right]$

and

$\tau_{\perp} \sim 4.5 \times 10^{3} \mathrm{~s} R_{16}\left[\epsilon \varphi^{1 / 3} R_{16}^{1 / 9} \Gamma^{-1 / 3} L_{38.5}^{1 / 9} n_{\mathrm{ISM}}^{1 / 18}\right]^{-1}$.

The adiabatic loss timescale is simply

$\tau_{\mathrm{ad}}=4 \frac{p_{\mathrm{c}}}{\dot{p}_{\mathrm{c}}} \sim \frac{R_{\mathrm{c}}}{\dot{R}_{\mathrm{c}}} \sim 7 \times 10^{5} \mathrm{~s} R_{16}^{5 / 3} n_{\mathrm{ISM}}^{1 / 3} L_{38.5}^{-1 / 3}$.

Since this is proportional to $R^{5 / 3}$, while $T_{\|} \propto R^{10 / 9}$ and $\tau_{\perp} \propto$ $R^{8 / 9}$, the particles are more likely to escape out of large, old cocoons than out of small, young ones.

\section{B.3. Summary}

The diffusion of CRs out of radio lobes is clearly an important problem not only in the context of CR emission from microquasars, but also for extragalactic radio sources and their contribution to the CR flux in clusters of galaxies. Since the current level of understanding is still very rudimentary, further study of this aspect is necessary. Given the large uncertainties in these estimates, and given that we tried to provide conservative estimates whenever possible, we feel that there is a good chance that the mechanisms outlined in this paper will work under realistic circumstances and that a measurable CR contribution from microquasars can be expected.

\section{References}

Achterberg, A., Gallant, Y. A., Kirk, J. G., \& Guthmann, A. W. 2001, MNRAS, 328, 393

Barrau, A. 2001 [astro-ph/0103493]

Bell, A. R. 1978, MNRAS, 182, 147

Berezhko, E. G., \& Ellison, D. C. 1999, ApJ, 526, 385

Binns, W. R., Wiedenbeck, M. E., Christian, E. R., et al. 2001, Adv. Space Res., 27, 767

Blandford, R., \& Eichler, D. 1987, Phys. Rep., 154, 1

Blandford, R. D., \& McKee, C. F. 1976, Phys. Fluids, 19, 1130

Blandford, R. D., \& Ostriker, J. P. 1978, ApJ, 221, L29

Boezio, M., Carlson, P., Francke, T., et al. 1999, ApJ, 518, 457

Borione, A., Chantell, M. C., Covault, C. E., et al. 1997, Phys. Rev. D, 55,1714

Castor, J., McCray, R., \& Weaver, R. 1975, ApJ, 200, L107

Dar, A., \& De Rújula, A. 2001, ApJ, 547, L33

Eikenberry, S. S., Matthews, K., Morgan, E. H., Remillard, R. A., \& Nelson, R. W. 1998, ApJ, 494, L61

Ellison, D. C., \& Double, G. P. 2002, Astropart. Phys., accepted [astro-ph/0202106]

Engelmann, J. J., Ferrando, P., Soutoul, A., Goret, P., \& Juliusson, E. 1990, A\&A, 233, 96

Ensslin, T., \& Sunyaev, R. A. 2001, A\&A, submitted

Ensslin, T. A., Biermann, P. L., Klein, U., \& Kohle, S. 1998, A\&A, 332, 395

Fender, R. P. 2001, MNRAS, 322, 31

Fender, R. P., Garrington, S. T., McKay, D. J., et al. 1999a, MNRAS, 304, 865

Fender, R. P., Hanson, M. M., \& Pooley, G. G. 1999b, MNRAS, 308, 473

Fender, R. P., \& Kuulkers, E. 2001, MNRAS, 324, 923

Fender, R. P., \& Pooley, G. G. 2000, MNRAS, 318, L1

Foster, R. S., Waltman, E. B., Tavani, M., et al. 1996, ApJ, 467, L81

Ginzburg, V. L. 1979, Theoretical physics and astrophysics (Pergamon), 457

Ginzburg, V. L. 1996, Phys.-Uspekhi, 39, 155

Han, X., \& Hjellming, R. M. 1992, ApJ, 400, 304

Haynes, R. F., Jauncey, D. L., Murdin, P. G., et al. 1978, MNRAS, 185,661

Heinz, S. 2002, A\&A, 388, L40

Hjellming, R. M., \& Rupen, M. P. 1995, Nature, 375, 464

Hunter, S. D., Bertsch, D. L., Catelli, J. R., et al. 1997, ApJ, 481, 205

Israelian, G., Rebolo, R., Basri, G., Casares, J., \& Martin, E. L. 1999, Nature, 401, 142

Kaiser, C. R., Sunyaev, R., \& Spruit, H. C. 2000, A\&A, 356, 975

Kennel, C., \& Petschek, H. E. 1966, J. Geophys. Res., 71, 1 
Kirk, J. G., \& Schneider, P. 1987, ApJ, 315, 425

Kotani, T., Kawai, N., Matsuoka, M., \& Brinkmann, W. 1998, in The Hot Universe, IAU Symp., 188, 358

Krymskii, G. F. 1977, Akademiia Nauk SSSR Doklady, 234, 1306

Labrador, A. W., \& Mewaldt, R. A. 1997, ApJ, 480, 371

Lloyd-Evans, J., Coy, R. N., Lambert, A., et al. 1983, Nature, 305, 784

Lubow, S. H., Papaloizou, J. C. B., \& Pringle, J. E. 1994, MNRAS, 267, 235

Manchanda, R. K., Waldron, L. E., \& Sood, R. K. 1993, Proc. Astron. Soc. Austr., 10, 208

Margon, B. 1984, ARA\&A, 22, 507

Marshall, H. L., Canizares, C. R., \& Schulz, N. S. 2002, ApJ, 564, 941

Martí, J., Mereghetti, S., Chaty, S., et al. 1998, A\&A, 338, L95

Menn, W., Hof, M., Reimer, O., et al. 2000, ApJ, 533, 281

Milgrom, M. 1979, A\&A, 76, L3

Milgrom, M., Anderson, S. F., \& Margon, B. 1982, ApJ, 256, 222

Mioduszewski, A. J., Rupen, M. P., Hjellming, R. M., Pooley, G. G., \& Waltman, E. B. 2001, ApJ, 553, 766

Mirabel, I. F., \& Rodríguez, L. F. 1994, Nature, 371, 46

Mirabel, I. F., \& Rodríguez, L. F. 1999, ARA\&A, 37, 409

Mirabel, I. F., Rodriguez, L. F., Chaty, S., et al. 1996, ApJ, 472, L111

Mirabel, I. F., Rodríguez, L. F., Cordier, B., Paul, J., \& Lebrun, F. 1992, Nature, 358, 215

Mitra, A. 1994, ApJ, 425, 782

Moskalenko, I. V., \& Strong, A. W. 1998, ApJ, 493, 694
Ogley, R. N., Bell Burnell, S. J., Spencer, R. E., et al. 2001, MNRAS, 326,349

Orosz, J. A., Kuulkers, E., van der Klis, M., et al. 2001, ApJ, 555, 489

Parker, E. N. 1965, Planet. Space Sci., 13, 9

Pooley, G. G., \& Fender, R. P. 1997, MNRAS, 292, 925

Pozdnyakov, L. A., Sobol, I. M., \& Syunyaev, R. A. 1983, Astrophys. Space Phys. Rev., 2, 189

Purcell, W. R., Cheng, L.-X., Dixon, D. D., et al. 1997, ApJ, 491, 725

Sams, B. J., Eckart, A., \& Sunyaev, R. 1996, Nature, 382, 47

Spencer, R. E. 1984, MNRAS, 209, 869

Stewart, R. T., Caswell, J. L., Haynes, R. F., \& Nelson, G. J. 1993, MNRAS, 261, 593

Strong, A. W., \& Moskalenko, I. V. 2001, in Proc. 27th Intern. Cosmic Ray Conf. (Hamburg, Germany), 27, 1942 [astro-ph/0106504]

Strong, A. W., Moskalenko, I. V., \& Reimer, O. 2000, ApJ, 537, 763

van Kerkwijk, M. H., Charles, P. A., Geballe, T. R., et al. 1992, Nature, 355,703

van Kerkwijk, M. H., Geballe, T. R., King, D. L., van der Klis, M., \& van Paradijs, J. 1996, A\&A, 314, 521

Vietri, M. 1995, ApJ, 453, 883

Yanasak, N. E., Wiedenbeck, M. E., Mewaldt, R. A., et al. 2001, ApJ, 563, 768

Zombeck, M. V. 1990, Handbook of space astronomy and astrophysics (Cambridge: University Press, 1990, 2nd ed.), 223 